\title{
1 Entamoeba histolytica EHD1 is involved in mitosome-endosome contact
}

2 Herbert J. Santos ${ }^{a, b}$, Yuki Hanadate ${ }^{b}$, Kenichiro Imai $^{c}$, Haruo Watanabe $^{d}$, and Tomoyoshi

3 Nozakia,b*

4 aDepartment of Biomedical Chemistry, Graduate School of Medicine, The University of

5 Tokyo, 7-3-1 Hongo, Bunkyo-ku, Tokyo 113-0033, Japan;

6 bDepartment of Parasitology, National Institute of Infectious Diseases, 1-23-1 Toyama,

7 Shinjuku-ku, Tokyo 162-8640, Japan;

$8 \quad{ }^{\mathrm{C}}$ Cellular and Molecular Biotechnology Research Institute, National Institute of Advanced

9 Industrial Science and Technology (AIST), 2-4-7 Aomi, Koto-ku, Tokyo 135-0064, Japan;

10 dDepartment of Bacteriology, National Institute of Infectious Diseases, 1-23-1 Toyama,

11 Shinjuku, Tokyo 162-8640, Japan

13 * Correspondence: nozaki@m.u-tokyo.ac.jp

\section{Abstract}

Inter-organellar crosstalk is often mediated by membrane contact sites (MCSs),

17 which are zones where participating membranes come within a proximity of $30 \mathrm{~nm}$. MCSs

18 have been found in organelles including the endoplasmic reticulum, Golgi bodies,

19 endosomes, and mitochondria. Despite its seeming ubiquity, reports of MCS involving

20 mitochondrion-related organelles (MROs) present in a few anaerobic parasitic protozoa

21 remain lacking. Entamoeba histolytica, the etiological agent of amoebiasis, possesses an

22 MRO called mitosome. We previously discovered several Entamoeba-specific

23 transmembrane mitosomal proteins (ETMPs) from in silico and cell biological analyses. 
24 One of them, ETMP1 (EHI_175060), was predicted to have one transmembrane domain

25 and two coiled-coil regions, and was demonstrated to be mitosome membrane-integrated

26 based on carbonate fractionation and immunoelectron microscopy (IEM)

27 data. Immunoprecipitation analysis detected a candidate interacting partner, EH-domain

28 containing protein (EHD1, EHI_105270). We expressed HA-tagged EHD1 in E. histolytica

29 and subsequent immunofluorescence and IEM data indicated an unprecedented MCS

30 between the mitosome and the endosome. Live imaging of GFP-EHD1 expressing strain

31 demonstrated that EHD1 is involved in early endosome formation, and is observed in

32 MCS between endosomes of various sizes. In vitro assay using recombinant His-EHD1

33 demonstrated ATPase activity. MCSs are involved in lipid transfer, ion homeostasis,

34 and organelle dynamics. The serendipitous discovery of the ETMP1 interacting partner

35 EHD1, led to the observation of the mitosome-endosome contact site in E. histolytica. It

36 opened a new view of how the relic mitochondria of Entamoeba may likewise be involved

37 in organelle crosstalk, a conserved feature of mitochondria and other organelles in

38 general.

Abstract word count: 248 words

\section{Introduction}

Membrane contact sites (MCSs) mediate communication and exchanges between

43 membrane-bound compartments by the assembly of protein-protein or protein-lipid

44 tethers which maintains distancing of $30 \mathrm{~nm}$ between interacting membranes. MCSs have

45 been found in almost every pair of organelles (1), most of which involving the endoplasmic

46 reticulum $(E R)$ as its membrane spans a network that interacts with the plasma 
47 membrane, and other organellar membranes such as those of the Golgi apparatus, lysosomes, endosomes, lipid droplets, peroxisomes, and mitochondria (2). MCSs are also reported between other organelle pairs such including the peroxisomes and lipid droplets,

50 and the mitochondria and vacuoles/endosomes/lysosomes, plasma membrane, lipid

51 droplets, and peroxisomes respectively, notwithstanding the contact sites between the

52 inner and outer mitochondrial membranes (3). These contact sites mostly harbor proteins

53 involved in lipid metabolism and transport, making them hubs of lipid transfer between

54 interacting membranes. However, other processes associated with MCSs have been

55 reported and they include ion transport and homeostasis, apoptosis (1), and endosomal

56 (4) and mitochondrial (5) fission, respectively.

We recently identified several key molecules that facilitate membrane contact sites in mitosomes, endosomes, and Golgi apparatus of Entamoeba histolytica, and aimed to

59 study their roles and possible link to the parasitic nature of this amoeba. E. histolytica is an anaerobic unicellular protozoan parasite that infects the large intestine of humans, and causes amebiasis, a disease characterized by diarrhea, which is a major cause of death

62 in children worldwide. Millions of individuals are infected; mostly in developing countries

63 and the disease causes an estimated 100,000 deaths annually (6). Infection begins by

64 the ingestion of infectious cysts, which are resistant to the acidic environment of the 65 stomach, then cysts pass through the small intestine, and undergo excystation within the 66 terminal ileum or colon, to the trophozoite stage. Trophozoites reproduce and encyst 67 within the colon, where they are released in the environment via excretion of feces, thus 68 completing one cycle of the fecal-oral transmission (Stanley, 2003). Invasive amoebic 69 trophozoites destroy the muco-epithelial barrier of the host intestinal tract, inducing mucus 
70 overproduction, inflammation and dysentery. It could lead to the formation of extra-

71 intestinal abscesses particularly in the liver (amoebic liver abscess), lungs, and brain. The

72 virulence of this parasite is due to its ability to inflict damage to host cells and tissues, via

73 parasite attachment to colonic epithelial cells, protease secretion to damage host cells

74 and evade host immune response, and by ingestion of host cells via phagocytosis and

75 trogocytosis. These processes involve intracellular trafficking and inter-organellar

76 crosstalk, underscoring the role of vesicular transport and membrane contact sites

77 (MCSs) not only in parasite biology but also in its virulence and pathogenesis.

78 Like other anaerobic parasitic protozoans, E. histolytica lacks canonical

79 mitochondria, and instead has a highly divergent mitochondrion-related organelle (MRO)

80 called mitosome. Entamoeba mitosomes contribute to parasitism (7) due to a

81 compartmentalized sulfate activation pathway that leads to the formation of cholesteryl

82 sulfate in the cytosol. This molecule induces stage conversion from the trophozoite to cyst

83 form (8) a process that is essential for maintaining the parasite's life cycle and mode of

84 disease transmission. Apart from mitosomes, other amoebic organelles such as the ER

85 and the Golgi apparatus also show less defined structural and compositional features

86 compared with model organisms, however, they have been shown to contain orthologs

87 of established endomembrane proteins (9-11). Our knowledge on MCSs in Entamoeba

88 is extremely limited, with only the previously identified mitosomal membrane proteins

89 ETMP30 (reported to interact with a Golgi-localized protein secretory pathway calcium

90 ATPase) and EHI_099350 (reported to have dual localization in the mitosomes and the

91 ER) (12-14). Other molecules that participate in tethering of these compartments and

92 what roles these contact sites play in the cell are still unknown, making it imperative to 
93 dissect amoebic MCSs. These past observations point to the fact that mitosomes,

94 although highly degenerate, are able to interact with other organelles in the cytoplasm,

95 and such contacts often utilize lineage-specific membrane. Here we identified another

96 mitosomal membrane protein, ETMP1 which interacts with a C-terminal Eps15 homology

97 domain (EHD) containing protein, a member of the EHD protein superfamily involved in

98 various endocytic processes. Studies on E. histolytica organelle interaction via the

99 endocytic transport mechanism has accumulated over several decades, including

100 proteins involved in cargo sorting regulation and endosome dynamics such as Rab

101 GTPases (15) and endosomal sorting complex required for transport (ESCRT) proteins

$102(16,17)$. However, there are so far no reports on whether these molecules take part in

103 MCS between endosomes and other parts of the cell.

105 Results

106 114 EHI_175060 with that of its orthologs in other Entamoeba species (E. moshkovskii, E.

\section{EHI_175060 is a lineage-specific mitosomal membrane protein}

Our group previously searched for transmembrane domain-containing mitosomal proteins using a previously-developed prediction pipeline (12) in sought for proteins that could be lineage-specific receptors, channels, enzymes, and components of the import machinery or otherwise uncharacterized complexes on the outer and inner membranes of Entamoeba histolytica mitosomes. This resulted in the prediction of 25 protein candidates. Like the other 24 proteins in the list (12), EHI_175060 is unique to the lineage Entamoeba. Figure 1 shows a multiple sequence alignment of the protein sequence of

115 dispar, and E. nuttalli). The protein has a predicted molecular mass of $29.5 \mathrm{kDa}$, and it 
116 contains two coiled-coil domains at the middle portion and a single transmembrane

117 domain near the carboxyl terminus. It also lacks a predictable canonical N-terminal

118 targeting sequence. Based on these characteristics, we name EHI_175060, as

119 Entamoeba-specific transmembrane mitosomal protein 1 (ETMP1).

120

121

122

123

124

125

126

127

128

129

130

131

132

133

134

135 those that contain chaperonin 60 (Cpn60; EHI_178570; a chaperone protein and 136 canonical mitochondrial matrix marker). The co-fractionation of HA-ETMP1 to mitosomes

137 was suggested by the anti-HA and anti-Cpn60 immunoblots of both the first and second 138 ultracentrifugation respectively (Figure 2C). We also performed subcellular fractionation 
139 followed by carbonate treatment, to further assess the localization, as well as membrane

140 integration of HA-ETMP1. The fractionation profile of HA-ETMP1 after immunoblot

141 analysis (Figure 2D, upper panel) showed that it is present in both cytosolic and organelle

142 fractions. Also, it was clearly demonstrated that the HA-ETMP1 contained in the

143 organellar membrane-enriched fraction, is integrated to organellar membranes as it was

144 retained in the particulate fraction after carbonate treatment, similar to MBOMP30-HA

145 (Figure 2D, middle panel), a positive control for mitosomal membrane protein. These

146 carbonate fractionation profiles contrast with that of the soluble mitosomal matrix protein

147 marker Cpn60, as shown by the blot stained with anti-Cpn60 antiserum (Figure 2D,

148 bottom panel).

149 We also performed immunoelectron microscopy analysis, and the results indicated

150 that HA-ETMP1 is localized to the mitosome membranes, as anti-HA gold particles are

151 found along the periphery of the Cpn60-labeled mitosomes (Figure 2E). Particle

152 distribution analysis of the gold-conjugated antibodies revealed a significant difference in

153 the staining of mitosomes $\left(368 \pm 279 / \mu \mathrm{m}^{2}\right)$ compared to cytosol $\left(22.3 \pm 9.25 / \mu \mathrm{m}^{2}\right)$ by

154 anti-HA gold. The distribution of the mitosomal marker APSK as detected by the gold anti-

155 APSK particles was also significantly higher in mitosomes $\left(192 \pm 98.1 / \mu \mathrm{m}^{2}\right)$ than in the

156 cytosol $\left(0.984 \pm 0.817 / \mu \mathrm{m}^{2}\right)$. Statistical significance in both datasets was analyzed using

157 two-tailed Welch's unequal variance t-test $(\mathrm{n}=17, p<0.0001)$. Overall, these data

158 provide evidence of mitosomal membrane localization of ETMP1.

159 ETMP1 is essential and its overexpression causes drastic growth defect

160 We made several attempts at silencing the etmp1 gene by small-RNA

161 transcriptional interference, all of which failed as the transformants did not survive drug 
162 selection, suggesting its essentiality to the parasite. We also observed a slower growth

163 rate in HA-ETMP1 expressors compared with mock control. Analysis of growth kinetics

164 of the two strains at varying concentrations of geneticin (G418) suggest a dose-

165 dependent effect of drug concentration to the growth of amebic trophozoites (Figure 3A),

166 and protein expression respectively (Figure 3B).

\section{ETMP1 interacts with EH-domain containing proteins}

To shed light on the function of ETMP1, we next attempted to identify its interacting partner(s) by immunoprecipitation (IP). Anti-HA agarose beads were used to immunoprecipitate the bait protein together with its binding partner(s) from the organelle-

171 rich fraction of HA-ETMP1-expressing and mock control strains respectively. Western

172 blotting with anti-HA antibody confirmed successful binding to and elution from HA173 ETMP1 with respect to the anti-HA beads (Figure 4A). Silver staining of the SDS-PAGE

174 gel containing HA peptide-eluted fractions revealed a band corresponding to 175 approximately $55 \mathrm{kDa}$ that is uniquely precipitated in HA-ETMP1 (absent in the mock-HA 176 control) (Figure 4B). Protein sequencing analysis by mass spectrometry followed by 177 differential comparison of quantitative values (QVs), normalized with unweighted 178 spectrum counts between HA-ETMP1 and mock-HA control identified interacting partners 179 of ETMP1 (Figure 4C). A cutoff of QV >2.0 in the HA-ETMP1 over the mock-HA sample 180 was used, yielded four candidates, three of which were exclusively detected in the eluted 181 IP fraction of HA-ETMP1. Also, three of the four candidates were identified in the 182 mitosome proteome that was previously published (18), namely, L-myo-inositol-1183 phosphate synthase (EHI_070720), EH-domain (EHD) containing protein 1 (annotated as 
184 receptor mediated endocytosis protein; EHI_105270) and its close homolog, EHD2 185 (EHI_152680).

We also performed Blue Native (BN)-PAGE analysis to assess whether ETMP1 is

187 part of a protein complex. Anti-HA immunoblot analysis of BN-PAGE run samples

188 indicated that HA-ETMP1 forms complexes of about $90 \mathrm{kDa}$ and $180 \mathrm{kDa}$ respectively

189 (Figure 4D). Protein sequencing analysis of the excised silver-stained BN-PAGE bands

190 containing these two complexes identified numerous proteins. Similarly, we set a cutoff

191 value of $>2.0$ and the list of proteins are found in Supplementary Table S1A-C. Notably,

192 EHD1 and its close homolog EHD3 (97\% identical) were identified in both the 90 and 180

193 kDa complex bands. Thus, we regarded EHD1 as one of the potential interacting partners

194 of ETMP1.

EHD1 is an ETMP1-interacting protein that is localized to mitosomes and to

vesicles of varying sizes

We expressed EHD1 in amoeba trophozoites with HA-tag at the amino terminus, as confirmed by the anti-HA immunoblot result showing a band corresponding to the expected molecular mass of HA-EHD1 ( 61 kDa) (Figure 5A). To analyze and confirm the mitosomal localization of EHD1, we performed double-staining IFA on HA-EHD1 expressing strain with anti-HA antibody and anti-APSK antiserum. We observed that the anti-HA signal is mostly localized to the membrane of vesicles of various sizes (Figure

203 5B). We could also notice a few punctate anti-HA signals which colocalized with the anti204 APSK mitosome marker (Figure 5C, white arrowheads). Although minimal colocalization 205 between anti-HA and anti-APSK signals were observed, some anti-APSK signals were 206 notably seen near the vesicle membranes marked with HA-EHD1 (Figure 5C, white 
207 arrow). Immunoelectron analysis (Figure 5D) corroborate the IFA observations, as we observed mostly vesicular membrane staining of anti-HA gold particles (left panel), with occasional signals on mitosomal membranes (middle panel), some of which showed

210 close proximity to vesicular membranes (right panel). Furthermore, immunoblot analysis

211 of Percoll-gradient fractions indicated wide distribution of HA-EHD1 across various

212 densities, mostly in fractions 9 to 10 and with weaker intensity in fractions 12 to 22 of the

213 first ultracentrifugation, and in fractions $\mathrm{A}$ to $\mathrm{N}$ in the second ultracentrifugation (Figure

$2145 \mathrm{E})$, validating the microscopic observations of HA-EHD1 vesicular and mitosomal 215 localization.

As majority of the signals of HA-EHD1 appear on vesicles, we next characterized

217 the vesicles marked by HA-EHD1 by performing co-staining IFA using anti-HA antibody

218 and one of the following antisera respectively: anti-vacuolar protein sorting 26 (Vps26),

219 anti-pyridine nucleotide transhydrogenase (PNT), and anti-Rab11B. Most of the anti-HA-

220 stained vesicles were colocalized with anti-Vps26- rather than anti-PNT- and anti-

221 Rab11B-stained vesicles (Figure 6A) as supported by the Pearson correlation $\mathrm{R}$ value

222 ranges of 0.22 to 0.37 for anti-Vps26, -0.16 to 0.19 for anti-PNT and 0.12 to 0.01 for anti-

223 Rab11B, respectively. Vps26 is a retromer complex component and is a marker of

224 endosomes/phagosomes in E. histolytica $(19,20)$. PNT is localized to the membrane of

225 numerous vesicles/vacuoles, including lysosomes and phagosomes (21), while Rab11B

226 was demonstrated to partially colocalize with late endosomes (22). Together, these data

227 suggest EHD1 is mostly localized to endosomal membranes which may contain Vps26

228 and to some extent PNT, but not Rab11B. 
HA-EHD1 is weakly associated to organellar membranes and preferentially binds

We performed a similar carbonate fractionation assay to the organelle-enriched

232 fraction of HA-EHD1 expressing strain. Based on the anti-HA immunoblots, HA-EHD1

233 was exclusively contained in the organelle fraction, as compared to that of the anti-CS1

234 profile which represents cytosolic fraction (Figure 6B). Next, we also assessed membrane

235 integration of HA-EHD1 by carbonate treatment of the organelle-enriched fraction.

236 Results of the immunoblots showed that HA-EHD1 is not membrane-bound as compared

237 to the lysosomal membrane protein marker CPBF1 (Figure 6B). Instead, the profile is

238 similar to that of the blot immunostained with an antiserum targeting Sec13, a peripheral

239 ER membrane protein (Figure 6B). This suggests that HA-EHD1 is not organellar

240 membrane-integrated, rather is weakly organellar membrane-associated.

To validate and characterize the phospholipid binding capacity of EHD1, we

242 carried out a lipid overlay assay using lysates of HA-EHD1 and HA-SNX1 (PI3P binding

243 protein control) respectively. Results indicated preferential binding of HA-EHD1 to

244 phosphoinositide diphosphates, specifically $\mathrm{PI}(3,5) \mathrm{P} 2$ and $\mathrm{PI}(4,5) \mathrm{P} 2$ (Figure 6C).

Overexpression of HA-EHD1 demonstrated enhanced multivesicular body (MVB)

246 formation

We also expressed HA-EHD1 under the control of tetracycline (tet) induction. IFA

248 analysis of HA-EHD1 showed the protein is similarly localized to membranes of various

249 vesicles after $1 \mathrm{~h}$ and $3 \mathrm{~h}$ of tet induced expression respectively (Figure 7A upper and

250 middle panel). However, at $24 \mathrm{~h}$ post induction with tet, we noticed drastic changes in the

251 localization as well as in the overall intracellular vesicular patterns of expressing 
252 trophozoites (Figure 7A bottom panel), wherein large multivesicular bodies (MVBs) that

253 were also marked with anti-HA signal were observed (Supplementary Movie S1). These

254 findings were also supported by immunoelectron micrographs, showing

255 immunodecoration of gold anti-HA particles along the membranes of MVBs, including the

256 neck of invaginated vesicles (Figure 7B), after $24 \mathrm{~h}$ of tet-induced expression of HA-EHD1.

257 These data point to the involvement of EHD1 in the biogenesis of MVBs in E. histolytica.

258 EHD1 is involved in early endosome formation during macropinocytosis and

259 receptor-mediated endocytosis

260 To further characterize the vesicles whose membranes are associated with EHD1,

261 we performed endocytosis assay using either dextran conjugated to rhodamine B

262 isothiocyanate (RITC) (for bulk endocytosis and macropinocytosis) as well as transferrin

263 conjugated to Alexa Fluor-568 (for receptor-mediated endocytosis) respectively, as

264 substrates, which were chased by live (GFP-EHD1 and mock-GFP) or fixed (HA-EHD1

265 and mock-HA) imaging analysis of treated strains. Expression of GFP-EHD1 was

266 confirmed as a single band after anti-GFP immunoblotting (Figure 8A). From imaging of

267 live GFP-EHD1, we observed that the GFP signals are evenly spread on round

268 endosomal membranes (Figure 8B left panel). However, signal polarization occurs on

269 portions where there is contact between two endosomes (Supplementary Movie S2). We

270 also observed localization of GFP-EHD1 in endosomes that contain either RITC-dextran

271 and Alexa Fluor 568-transferrin (Figure 8B middle and right panels respectively). Our

272 observations also revealed that EHD1 is involved in early endosome formation during

273 macropinocytosis of RITC-dextran. Membranes of newly formed vesicles after ingestion

274 of RITC-dextran initially did not contain EHD1 but several seconds later, GFP-EHD1 
275 showed intense signal on the membrane of the enclosing early endosome

276 (Supplementary Movie S3). Consistent with this, we also noticed a similar phenomenon

277 of GFP-EHD1 recruitment in closing early endosomes when Alexa Fluor-568-transferrin

278 was used as substrate (Supplementary Movie S4). In addition, we observed accumulation

279 of transferrin on to certain spots in the plasma membrane which showed remarkably high

280 GFP-EHD1 signal (Supplementary Movie S5). This suggests that EHD1 is also involved

281 in intra-vesicular traffic of transferrin with some aggregate signals localized near the PM,

282 likely hinting at its involvement in receptor or membrane recycling.

283

284

285

286

287

288

289

290

291

292

293

294

295

296

297

\section{HA-EHD1 is localized to phagosome and trogosome membrane}

To further characterize EHD1-containing vesicles, we performed phagocytosis assay by co-incubating expressing trophozoites with CellTracker Blue-stained Chinese hamster ovary $(\mathrm{CHO})$ cells. Live and fixed-cell imaging analyses of phagosomes or trogosomes containing whole, or bites of $\mathrm{CHO}$ cells, respectively were observed at varying time points after co-incubation. We observed association of either GFP-EHD1 or HA-EHD1 on some phagosome and trogosome membranes (Figure 9). We also noticed patches of higher intensity signals on certain regions of contact between phago- or trogosomes and other vesicles in both fixed (Supplementary Movie S6) and live (Supplementary Movie S7) cell imaging analyses. IFA images also suggest that HA-EHD1

is localized at the phagocytic cup/tunnel suggesting its involvement in early phagosome formation (Figure 9 top panel; 15 min post coincubation). Also observed in fixed cells was the localization of HA-EHD1 on the trogosome membrane that appears to undergo tubulation (Figure 9, bottom panel; 60 min post co-incubation).

\section{Recombinant His-EHD1 demonstrated ATPase activity in vitro}


We also expressed amino terminus histidine (His)-tagged E. histolytica EHD1 in bacteria to assess its enzymatic activity in vitro. We purified His-EHD1 using nickelnitriloacetic acid (Ni-NTA)-agarose beads as shown by the Coomassie Brilliant Bluestained SDS-PAGE gel, as well as the anti-His antibody-stained PVDF membrane (Figure 10A), containing representative Ni-NTA purification fractions. Eluted fraction of purified

303 His-EHD1 demonstrated ATPase activity (Figure 10B) with a Michaelis-Menten constant $304(\mathrm{Km})$ value of $94.91 \pm 16.63 \mu \mathrm{M}$ and a maximum velocity $\left(\mathrm{V}_{\max }\right)$ of $9.85 \pm 0.37$ $305 \mu \mathrm{mole} / \mathrm{min} / \mathrm{mg}$.

\section{Discussion}

We have verified our prediction of ETMP1 being localized to the mitosomal membrane by imaging and fractionation analyses. The gene encoding for this protein is essential to the parasite's proliferation as indicated by the failure of transfected

311 trophozoites to survive sublethal concentration of drug pressure, compared with those

312 transfected with an empty vector control. Previous attempts at silencing the genes

313 encoding other mitosomal membrane proteins such as Tom40 (23) and MBOMP30 (24)

314 also failed, suggesting the essential role that these proteins, and the mitosome itself 315 where they exclusively localize, maintain in the proliferation of $E$. histolytica.

316 Overexpression of ETMP1 also affected the growth rate of the parasite negatively. This

317 may be due to the disruption of tight regulatory mechanisms for maintaining mitosomal 318 homeostasis and/or formation of toxic protein aggregates. It could also be due to the 319 stoichiometric imbalance of HA-ETMP1-containing protein complexes. Our BN-PAGE 320 analysis identified ETMP1 in the $90 \mathrm{kDa}$ and $180 \mathrm{kDa}$ complexes respectively, whose 
321 formation, compositional ratios, and biological functions may be sensitive to ETMP1 322 overexpression.

HA-ETMP1 immunoprecipitated a unique $55 \mathrm{kDa}$ protein. Mass spectrometry

324 analysis of the excised silver-stained gel band indicated several candidates including EH-

325 domain containing protein (EHD1; EHI_105270; 58 kDa) and its ortholog (EHD2;

326 EHI_152680; 58 kDa) sharing 82\% identity, vacuolar protein sorting-associated protein

32745 (60 kDa; EHI_154290), and L-myo-inositol-1-phosphate synthase (57 kDa;

328 EHI_070720). Incidentally, when we sequenced the complex band of 90 and 180 kDa

329 BN-PAGE complex bands that include HA-ETMP1, we identified EHD1 and its close

330 homolog EHD3 (EHI_052870; 58 kDa) with 97\% identity. From these data, we deduced

331 a plausible interaction between ETMP1 and EH domain containing proteins, with a focus

332 on EHD1 in this paper. Repeated multiple attempts at immunoprecipitating the said

333 complexes failed. One possibility is that the topology of HA-ETMP1 in the complex

334 blocked the HA epitope tag from binding to the anti-HA beads. We also performed IP

335 using HA-EHD1 (Supplementary Figure S1, Supplementary Table S2A), however our

336 protein sequencing analysis of the $\sim 30-37 \mathrm{kDa}$ excised band did not detect HA-ETMP1

337 (Supplementary Table S2B), suggesting the likely transient nature of this protein binding.

338 The detection of amoebic EHD isotypes in the pull-down and BN-PAGE complexes of

339 HA-ETMP1 respectively, suggests potential interaction among these EHD homologs. It is

340 also plausible that amoebic EHDs form hetero-dimers or hetero-oligomers as was

341 demonstrated by mouse EHD1 and EHD3. The interaction between mouse EHD1 and

342 EHD3 is likely involved in the regulation of recycling endosomes movement along

343 microtubules (25). In E. histolytica, such EHD oligomers may be involved not only during 
344 endocytosis but may also exist during the formation and maintenance of the mitosome-

345

346

347

348

349

350

351

352

353

354

355

356

357

endosome contact. Compositional variations of EHD homo- or hetero-oligomers may also exist, and their corresponding functions may be stoichiometry-dependent.

EHDs have been associated to play roles in various endocytic processes. In one subset known as the C-terminal EHDs, four paralogues are present in mammals, namely EHD1, EHD2, EHD3, and EHD4. Mammalian EHD1 regulates exit of proteins from the endocytic recycling compartment to the plasma membrane, while both EHD1 and EHD3 have similar roles in controlling early endosome to Golgi transport $(26,27)$. Mammalian EHD2 localizes to caveolae and together with the Bin Amphiphysin Rvs (BAR)-domain containing binding partner PACSIN2, stabilizes caveolae at the cell surface (28), whereas mammalian EHD4 facilitates macroendocytic uptake of tropomyosin receptor kinase (Trk) receptors (29). EHDs are also implicated in the regulation of endocytic pathways associated with lipid metabolism. Mammalian EHD1 is involved in cholesterol homeostasis, affecting generation of cholesterol and triglyceride lipid bodies (30).

EHDs also regulate endocytosis in other organisms including plants, worms, and protozoans. Arabidopsis thaliana has two EHD paralogs, AtEHD1 and AtEHD2. Downregulation of AtEHD1 led to a deficiency in the entry of endocytosed material into plant cells, whereas overexpression of AtEHD2 caused an inhibitory effect on endocytosis, suggesting both proteins are important components in plant endocytic machinery (31). The EHD ortholog in Caenorhabditis elegans, receptor-mediated endocytosis 1 (Rme1), localizes to the endocytic recycling compartment and mediates the exit of cargo proteins to the cell membrane (32). In the protozoan parasite that causes malaria, Plasmodium falciparum, a single EHD protein is encoded in its genome. PfEHD is involved in 
367 endocytosis and plays a role in the generation of endocytic vesicles at the plasma

368 membrane, that are subsequently targeted to the neutral lipid generation/storage site

369 localized near the food vacuole (33). In the free-living amoebozoan Dictyostelium

370 discoideum, a single gene encoding EHD protein was identified. DdEHD was determined

371 to be involved in phagosome maturation, and its deletion resulted to defects in

372 intraphagosomal proteolysis and acidification, early delivery of lysosomal enzymes, and

373 fast retrieval of the vacuolar $\mathrm{H}^{+}$-ATPase in maturing phagosomes (34).

We have shown that EhEHD1 is involved in various endocytic processes. Our live

375 imaging analysis showed involvement of EhEHD1 in early endosome formation,

376 particularly during closure of newly-formed endosomes after engulfment of either RITC-

377 dextran (Supplementary Movie S3) or Alex568-transferrin (Supplementary Movie S4),

378 suggesting that EhEHD1 may participate in the scission of early endosomes generated

379 from macropinocytosis as well as receptor-mediated endocytosis. Vesicle tubulation and

380 scission are associated roles of EHDs as they possess a dynamin-like ATPase domain

381 as demonstrated previously in (34-37).

382 Our in vitro enzyme assay showed that His-EHD1 has ATPase activity with a $\mathrm{Km}$

383 value of $94.91 \pm 16.63 \mu \mathrm{M}$ as compared to the previously reported $\mathrm{Km}$ values for mouse

384 EHD1 $(80 \mu \mathrm{M})$ and CeRME1 $(30-\mu \mathrm{M})(38)$. We also attempted to investigate the role of

385 ATPase activity of EHD1 in E. histolytica by expressing ATPase-deficient dominant

386 negative mutant, however the transfectants did not survive drug selection, suggesting the

387 importance of EHD1 ATP hydrolysis in amoebic biology. Based on other works, ATPase

388 activity of C-terminal EHD-containing proteins is crucial for various stages of the endocytic

389 traffic machinery. Hydrolysis of ATP was essential for binding of human EHD2 complexes 
390 to caveolae during clathrin-independent endocytosis (39). It is suggested that membrane

391 scission results from ATP hydrolysis by human EHD2 in vivo (35). Using cross-

392 complementation assays in C. elegans, ATP binding and hydrolysis of human EHD1 was

393 essential for endocytic recycling. It was also shown using in vitro liposome-based assays

394 that ATP binding of human EHD1 promote scaffold self-assembly, while ATP hydrolysis

395 enables extension of bulges and thinning of tubular model membranes which leads to

396 scission (40). In vitro analysis also revealed that ATP binding and concomitant hydrolysis

397 allows membrane remodelling into highly curved tubules (29). We can only hypothesize

398 that ATP hydrolysis in amoebic EHD1 may have functions similar to its homologs in other

399 organisms.

$400 \quad$ We also detected EhEHD1 in the phagocytic cup, and membranes of phagosomes

401 and trogosomes, although only a select few phagosomes and trogosomes are labeled

402 with EhEHD1 in both live and fixed imaging analysis. The same can also be said when

403 we performed endocytosis assay using either RITC-dextran or Alexa Fluor 568-transferrin.

404 This suggests the nature of EHD localization being dependent on either recruitment by

405 interacting proteins or association/binding with certain lipids on vesicular membranes at

406 specific time points. This is reflected by the localization of either GFP-EHD1 or HA-EHD1

407 in membranes of vesicles of various sizes, and the seemingly polarized signal intensity

408 onto sites where two vesicles are in close contact.

409 As suggested by our lipid overlay assay result, EhEHD1 preferentially binds to

$410 \mathrm{PI}(3,5) \mathrm{P} 2$ and $\mathrm{PI}(4,5) \mathrm{P} 2 . \mathrm{PI}(4,5) \mathrm{P}_{2}$ has been demonstrated to be localized to the plasma

411 membrane (41), lipid rafts and uroids (42) of E. histolytica. It is important to note that

$412 \mathrm{PI}(4,5) \mathrm{P} 2$ localized at the plasma membrane is involved initiating internalization during 
413 endocytosis, micropinocytosis, and phagocytosis $(43,44)$, whereas $\mathrm{Pl}(3,5) \mathrm{P} 2$ has a

414 critical role in endosome/lysosome biogenesis, and in the initiation of MVB formation (45).

415 Together, these results circumstantially support our observations of amoebic EHD1

416 localization and involvement in early endosome, intraluminal vesicle, and MVB formation.

417 On the possible role(s) of mitosome-endosome contact in E. histolytica, we posit

418 that this MCS may be involved in lipid transfer, ion transport, and quality control. Lipid

419 transport and/or metabolism are commonly alluded roles of MCSs. Although we did not

420 detect any lipid transport proteins in our immunoprecipitation assay, two lipid transport

421 proteins (LTP1 and LTP3) in E. histolytica have been characterized (46), and it is plausible

422 that various LTPs may transiently interact with amoebic MCSs to facilitate lipid mobility

423 across organelles. We detected a few fatty acid ligases in the $\sim 90$ and $\sim 180 \mathrm{kDa}$ complex,

424 however the interaction of these proteins to the HA-ETMP1 containing complex needs to

425 be experimentally validated. Alternatively, ion transport may also be facilitated in this MCS,

426 as was demonstrated in epithelial cells, where the mitochondria and endosomes that

427 contain iron-bound transferrin are involved in "kiss and run" interactions, leading to iron

428 transfer from endosomes to mitochondria (47). Another possibility is the involvement of

429 EHD1 in mitosomal dynamics. Mitochondria undergo dynamics of fusion and fission to

430 ensure maintenance of homeostasis, control of reactive oxygen species, apoptosis, and

431 autophagy. Dynamin and dynamin related proteins (Drps) have been implicated in

432 mitochondrial fission. Recently in HeLa cells, EHD1 was reported to be a novel regulator

433 of mitochondrial fission via a mechanism distinct from that of dynamin/Drp. In this model

434 human EHD1, together with its binding partner rabankyrin-5 interact with the retromer

435 complex participate in mitochondrial division. EHD1 was suggested to facilitate the fission 
436 of vesicles that transport Vps35, a retromer complex component, from endosomes to the

437 mitochondrial membrane. It was also suggested that Vps35 may interact with inactive

438 Drp1 on the mitochondrial membrane, causing its removal and subsequent action of

439 active Drp1 to perform mitochondrial fission (48). Fission has also been reported in MROs

440 of anaerobic parasites such as the hydrogenosomes of Trichomonas vaginalis and $E$.

441 histolytica (49-51). Mitosome fission in E. histolytica was reported to involve a

442 heterodimer complex of two dynamin-related proteins, DrpA and DrpB (50). It is

443 interesting if amoebic EHD1 also takes part in influencing mitosome fission as was

444 postulated in mammalian cells (48). An alternative novel pathway for mitochondrial quality

445 control that is independent of Atg5 and LC3 is the formation of mitochondria-derived

446 vesicles targeted to lysosomes. Ultrastructural analysis of COS7 cells identified the

447 presence vesicles that are Tom20-positive within MVBs (52). Furthermore, in hepatocytes,

448 a complex made up of EHD2, EH domain binding protein 1 (EHBP1), and Rab10,

449 promotes extension of the LC3-containing autophagic membrane in order to engulf lipid

450 droplets during lipophagy (53). Such related pathways may also exist in E. histolytica that

451 warrants further investigation in the future.

\section{Conclusion}

We report a novel membrane contact site between mitosomes and endosomes of

455 Entamoeba histolytica. This unprecedented MCS features the mitosomal membrane 456 protein ETMP1 and a C-terminal EH domain containing protein, EHD1. ETMP1 is a 457 protein unique to Entamoeba and is essential to parasite proliferation. It interacts with 458 EHD1, a protein involved in various endocytic processes in $E$. histolytica, namely in early 
459 endosome formation during bulk and receptor-mediated endocytosis, phagocytosis and

460 trogocytosis of mammalian cells, and in the invagination of intraluminal vesicles for the

461 generation of multivesicular bodies. Such novel ETMP1-EHD1 interaction hints at a

462 possible role of this mitosome-endosome MCS on various physiological processes that

463 have been demonstrated in other organisms. We thus propose that ETMP1-EHD1

464 mediated contact site is involved in lipid transfer, biogenesis, autophagy, organelle

465 dynamics and quality control of MROs. Further investigation is needed to fully dissect the

466 molecular mechanisms and functions of this and other MRO-related MCSs.

Materials and Methods

469 Entamoeba histolytica cultivation

470 Entamoeba histolytica HM-1:IMSS Cl6 (54) and G3 (55) strains were maintained

471 in Diamond's BI-S-33 medium (54) as described previously. Subculture was performed

472 after incubation of up to 3-4 days when trophozoites reached the late-logarithmic phase.

473 Plasmid construction

474 Extraction of total RNA from E. histolytica trophozoites, purification of mRNA, and

475 synthesis of cDNA were performed by following protocols described previously (24). For

476 the expression of hemagglutinin (HA) tagged proteins in E. histolytica trophozoites, target

477 genes (etmp1: EHI_175060 and ehd1: EHI_105270) were amplified by polymerase chain

478 reaction $(\mathrm{PCR})$ using E. histolytica cDNA as template, and the corresponding primer sets:

479 (etmp1-Xmal-fwd: GTTccogggATGGAACAAAAACTGAAGAA; etmp1-Xhol-rev:

480 GAActcgagTTATTTTTTCATTTTTCTTAAGG; and ehd1-Xmal-fwd:

481 GTTccogggATGTTTGGTAAGAAGAACAAAAACC; ehd1-Xhol-rev: 
482 GAActcgagTTATTCAACTGGTGGAAGATTGTC). These PCR amplicons were inserted

483 into the following plasmids: pEhEx-HA and pEhEx-GFP for constitutive expression, (56)

484 and pEhtEx-HA and pEhtEx-GFP for tetracycline-induced expression (50), after digestion

485 with Xmal and Xhol (New England Biolabs, Beverly, MA, USA) and then ligated using

486 Ligation Convenience Kit (Nippongene, Tokyo, Japan). For the expression of recombinant

487 proteins in Escherichia coli, PCR-amplification of ehd1 was performed using E. histolytica

488 cDNA as template and the following primer set (ehd1-BamHI-fwd:

489 GTTggatccATGTTTGGTAAGAAGAAACAAAAACC, and ehd1-Sall-rev:

490 GAAgtcgacTTATTCAACTGGTGGAAGATTGTC). Digestion and ligation to BamHI and

491 Sall-linearized pCold plasmid (Takara, Shiga, Japan) were performed. For transcriptional

492 gene silencing, about 400 bp fragments of etmp1 and ehd1 were amplified using cDNA

493 and appropriate primer sets: etmp1gs-Stul-fwd: GTTaggcttATGGAACAAATAACTGAAG;

494 etmp1gs-Sacl-rev: GAAgagctcCTAATTTGATTCCTTTTAAAG; and ehd1gs-Stul-fwd:

and

ehd1gs-Sacl-rev:

GAAgagctcTAAATTTAGCCATAAATTCAT. The amplicons were digested with Stul and

497 Sacl and ligated to pSAP2-Gunma (57).

Amoeba transfection and drug selection

The constructed plasmids described above were transfected by lipofection into $E$.

501 performed by changing the culture medium supplemented with G418 (Gibco/Life

502 Technologies, USA) for those transfected with pEhEx-based plasmids, or with

503 hygromycin (Fujifilm Wako, Japan) for those transfected with pEhtEx-based plasmids.

504 The starting concentration of either $1 \mu \mathrm{g} / \mathrm{mL}$ G418 or hygromycin added was gradually 
505 increased until all control cells (transfected without plasmid) died from the antibiotic

506 challenge. All resultant strains were maintained in medium containing $10 \mu \mathrm{g} / \mathrm{mL}$ G418 or

$50720 \mu \mathrm{g} / \mathrm{mL}$ hygromycin unless otherwise stated. For tetracycline-induction of protein

508 expression, $10 \mu \mathrm{g} / \mathrm{mL}$ tetracycline was added to semi-confluent cultures $24 \mathrm{~h}$ prior to

509 performing assays unless otherwise stated.

510 Immunoflourescence assay (IFA)

511 Double-staining immunofluorescence assay was performed as previously

512 described (13), using anti-HA mouse monoclonal antibody (clone 11MO, Covance, USA)

513 diluted $1: 500$ in $2 \%$ saponin and $0.1 \%$ bovine serum albumin in phosphate buffered saline

514 (saponin-BSA-PBS), to detect HA-tagged ETMP1 and EHD1, respectively, and one of the

515 following polyclonal rabbit antisera diluted 1:1000 in saponin-BSA-PBS unless otherwise

516 stated: anti-adenosine-5'-phosphosulfate kinase (APSK; EHI_179080; a mitosomal

517 matrix protein; (57) diluted 1:300; anti- vacuolar protein sorting 26 (Vps26; EHI_062490;

518 a retromer complex component (19)); anti-Rab11B (EHI_107250; involved in cysteine

519 protease secretion; (22)); and anti-pyridine nucleotide transhydrogenase (PNT;

520 EHI_014030; a novel class of lysosomal PNT; (21)). Secondary antibodies used were

521 Alexa Fluor-488 anti-mouse antibody and Alexa Fluor-568 anti-rabbit antibody Thermo

522 Fisher diluted 1:1000 in saponin-BSA-PBS, respectively. Cells were visualized using

523 LSM780 (Carl Zeiss Microscopy, Germany) confocal laser scanning microscope.

\section{Subcellular fractionation and immunoblot analysis}

525

Trophozoites at the late-logarithmic phase were collected and washed thrice with

$5262 \%$ glucose-PBS. Cells were mechanically disrupted using a Dounce homogenizer as

527 described previously (13). The resulting homogenate was separated by Percoll-gradient 
528 fractionation as previously described $(13,18)$. For carbonate fractionation, organelle-

529 enriched fractions from HA-ETMP1, MBOMP30-HA, HA-EHD1 and mock control

530 homogenates were collected by centrifugation at $100,000 \mathrm{~g}$ for $60 \mathrm{~min}$ at $4^{\circ} \mathrm{C}$. The

531 resultant pellet was reacted with sodium carbonate as previously described $(13,23,24)$.

532 All fractions collected were run in SDS-PAGE followed by Western blotting as previously

533 described (60). Immunostaining of PVDF membranes was performed using anti-HA 534 antibody, anti-APSK antiserum (organelle fraction marker), anti-cysteine synthase 1

535 (CS1; EHI_171750; cytosolic enzyme involved in cysteine metabolism) (61), anti-cysteine

536 protease binding family protein 1 (CPBF1; EHI_164800, membrane fraction control) (62)

537 and chemiluminescent bands were visualized using LAS-4000 mini luminescent image 538 analyzer (Fujifilm Life Science, Tokyo, Japan).

539 In silico predictions and analyses

$540 \quad$ Transmembrane domain-containing mitosomal proteins were predicted using a 541 pipeline developed in our previous study (12). To search for homologs of ETMP1 in

542 various Entamoeba species, we used as query the E. histolytica protein, EHI_175060, 543 and implemented a BLAST search using the Amoebozoa resource database, AmoebaDB 544 (63). Coiled-coil regions were predicted using DeepCoil (64).

\section{Immunoelectron microscopy}

$546 \quad$ Samples were prepared as described previously (24). The specimens were 547 double-stained with anti-HA mouse antibody, and anti-APSK rabbit antiserum (57). 548 Processing and visualization were performed by Tokai Microscopy Inc. (Nagoya, Japan), 549 using a transmission electron microscope (JEM-1400 Plus, JEOL Ltd., Japan) at an 
550 acceleration voltage of $80 \mathrm{kV}$. Digital images with a resolution of $2048 \times 2048$ pixels were

551 taken using a CCD camera (VELETA, Olympus Soft Imaging Solution GmbH, Germany).

552 Immunoprecipitation (IP) of HA-ETMP1 by anti-HA antibody

553 Organelle-enriched fractions from HA-ETMP1 and mock pEhEx-HA control

554 homogenates were prepared and approximately $2 \mu \mathrm{g}$ of proteins were solubilized in $2 \%$

555 digitonin in IP Buffer containing $50 \mathrm{mM}$ BisTris- $\mathrm{HCl}, \mathrm{pH} 7.2,50 \mathrm{mM} \mathrm{NaCl}, 0.001 \%$

556 Ponceau S, and $10 \% \mathrm{w} / \mathrm{v}$ glycerol for $30 \mathrm{~min}$ on ice. The solubilized fraction was collected

557 by centrifugation at $20,000 \mathrm{~g}$ for $30 \mathrm{~min}$ at $4{ }^{\circ} \mathrm{C}$. Immunoprecipitation was performed as

558 previously described (23). Bound proteins were eluted overnight using $60 \mu \mathrm{\mu g}$ HA peptide.

559 Eluted fractions were loaded on SDS-PAGE gels, followed by immunoblotting using 560 mouse anti-HA antibody. Silver staining was performed using the Silver Stain MS kit

561 (Fujifilm Wako Pure Chemical Corporation, Osaka, Japan), according to the

562 manufacturer's protocol. Protein sequencing by liquid chromatography-mass

563 spectrometry analysis was conducted by the Biomolecular Analysis Facility Core,

564 University of Virginia.

\section{Lipid overlay assay}

566 As described previously (20), the lysate of HA-EHD1 expressing strain was used 567 to probe a P-6001 phospholipid membrane strip (Echelon Biosciences, Salt Lake City, 568 Utah, USA). The lysate of HA-SNX1 which binds to PI3P (20) was used as a positive 569 control. The strips were washed three times with $0.1 \%$ Tween 20 in PBS (PBS-T), 570 followed by reaction with 1:1000 anti-HA mouse antibody in 3\% BSA-PBS for $2 \mathrm{~h}$ at room

571 temperature. The strips were washed and incubated with 1:6000 HRP-conjugated goat 572 anti-mouse IgG (Thermo-Fisher Scientific, USA) in 3\% BSA-PBS for $1 \mathrm{~h}$ at room 
573 temperature. Finally, the strips were washed and reacted with the Immobilon ECL Ultra

574 Western HRP Substrate (Millipore, USA) following manufacturer's instructions.

575 Endocytosis assay

576 Approximately $1 \times 10^{5}$ GFP-EHD1 or mock-GFP expressing trophozoites in $1 \mathrm{~mL}$

577 Bl-S-33 of strains respectively were placed on a $35 \mathrm{~mm}$ collagen-coated glass- bottom

578 culture dish (MatTek Corporation, Ashland, MA) for 15 minutes to allow for cell attachment.

579 The medium was removed and replaced with $1 \mathrm{~mL}$ of BI-S-33 supplemented with either

5802 mg/mL RITC-dextran (MW = 70 000; Sigma-Aldrich, USA) or 100 g/ml Alexa Fluor-

581568 transferrin (Thermo-Fisher Scientific, USA). Chase was performed for up to 30 min

582 for live imaging. For IFA, fixation was conducted onto HA-EHD1 and mock-HA strains

583 after $0,30,60,120$ min of addition of either RITC-dextran or Alexa Fluor 568-transferrin.

584 Images were captured using a confocal laser scanning microscope LSM780 (Carl Zeiss

585 Microscopy, Germany), as the cells were being incubated at $35^{\circ} \mathrm{C}$ using a temperature-

586 controlled stage plate (Carl Zeiss Microscopy, Germany).

\section{Phagocytosis assay}

588 A semi-confluent culture of Chinese hamster ovary $(\mathrm{CHO})$ cells, grown in F12 589 medium (Sigma-Aldrich, USA) was stained by addition of $40 \mu \mathrm{M}$ CellTracker Blue 590 (Thermo-Fisher Scientific, USA) for $30 \mathrm{~min}$ at $37^{\circ} \mathrm{C}$. The medium containing excess dye 591 was removed and the cells were washed in 1X PBS followed by treatment with $0.1 \%$ 592 trypsin for 5 min at $37^{\circ} \mathrm{C}$. The detached cells were collected and washed with $1 \mathrm{X}$ PBS 593 thrice by centrifugation at $3000 \mathrm{rpm}$ for 3 minutes. Stained $\mathrm{CHO}$ cells were resuspended 594 in BI-S-33 medium prior to addition to amoeba cells. Cells were co-incubated for 15, 30, 595 and 60 min respectively, after which they were fixed for IFA analysis as mentioned above, 
596 but using anti-GFP antibody (Sigma-Aldrich, USA) and anti-Vps26 antiserum respectively.

597 A parallel setup was prepared for live imaging analysis.

Expression and purification of recombinant His-EHD1

$601 \mu \mathrm{g} / \mathrm{ml}$ of ampicillin. Isolated colonies were cultured in LB medium with $150 \mu \mathrm{g} / \mathrm{ml}$ of 602 ampicillin, incubated at $37^{\circ} \mathrm{C}$ with shaking. A $1 \mathrm{~L}$ culture was inoculated and incubated in 603 a shaker at $37^{\circ} \mathrm{C}$ until reaching optical density (OD) 600 of 0.7 . The culture was flash 604 cooled in an ice water bath for 30 minutes. Induction of protein expression was made by 605 adding $0.5 \mathrm{mM}$ isopropyl $\beta$-D-thiogalactopyranoside (IPTG) to the medium followed by 606 incubation at $15^{\circ} \mathrm{C}$ with shaking for $24 \mathrm{~h}$. Cells were collected and protein expression 607 was confirmed by loading the soluble and insoluble fractions in SDS-PAGE followed by 608 Coomassie blue staining and anti-His immunoblot analysis respectively as described previously (65). His-EHD1 was purified by binding with $\mathrm{Ni}^{2+}-\mathrm{NTA}$ His-bind slurry (Qiagen,

610 Germany) and eluting with imidazole as described previously (65). Purified His-EHD1 was

611 stored at $-80^{\circ} \mathrm{C}$ with $20 \%$ glycerol in small aliquots until use.

\section{Enzyme activity assay}

Varying amounts of purified His-EHD1 $(0,0.125,0.25,0.5,1.0 \mu \mathrm{g})$ were

614 resuspended in assay buffer (20 mM HEPES pH 7.5, 0.005 \% Tween 20, $10 \%$ glycerol,

$6151 \mathrm{mM}$ DTT, $20 \mathrm{mM} \mathrm{NaCl}$, and $10 \mathrm{mM} \mathrm{MgCl}_{2}$ ) and loaded triplicate onto independent wells

616 of a 96-well plate. Then, $2 \mu \mathrm{l}$ of $100 \mathrm{mM}$ ATP was used as substrate and distilled water

617 was added to bring the volume of the mixture to $20 \mu$ l. Finally, $20 \mu$ l of $2 X$ stock solution

618 (66) containing $100 \mathrm{mM}$ Tris- $\mathrm{HCl}\left(\mathrm{pH}\right.$ 7.5), $10 \mathrm{mM} \mathrm{MgCl}_{2}, 0.02 \%$ Triton $\mathrm{X}-100,0.01 \%$ 
619 BSA, 2 mM glucose, 0.2 mM NADP, 2 u/mL ADP-hexokinase, 2 U/mL glucose-6620 phosphate dehydrogenase, $2 \mathrm{U} / \mathrm{mL}$ diaphorase I, $0.1 \mathrm{mM}$ resazurin in DMSO, and $20 \mathrm{mM}$

$621 \mathrm{~N}$-ethylmaleimide in DMSO, was added and the plate was incubated at $37^{\circ} \mathrm{C}$ for $30 \mathrm{~min}$.

622 For determining kinetic parameters, $0,12.5,25,50,75,100,125,250,500,750,1000$,

623 and $2500 \mu \mathrm{M}$ ATP was used to react with $1.5 \mu \mathrm{g}$ His-EHD1 for $30 \mathrm{~min}$. The fluorescence

624 was measured continuously at excitation and emission wavelengths of $540 \mathrm{~nm}$ and 625 590nm, respectively using SpectraMax Paradigm Multi-Mode microplate reader 626 (Molecular Devices, San Jose, CA, USA).

\section{Acknowledgments}

630 This research is funded by Grants-in-Aid for Scientific Research (B) (JP18H02650 and 631 JP21H02723 to T.N.), Grants-in-Aid for Young Scientists (JP20K16233 to H.J.S.) and 632 Core-to-Core Program, (JPJSCCB20190010) from the Japan Society for the Promotion 633 of Science, Grant for research on emerging and re-emerging infectious diseases from 634 Japan Agency for Medical Research and Development (AMED, JP20fk0108138 to T.N.).

635 The authors want to thank Mihoko Imada of The University of Tokyo for her techincal 636 support. The authors also want to thank Dr. Takashi Makiuchi of Tokai University School 637 of Medicine, Japan, and Dr. Eri Hayakawa of Jichi Medical University, Japan for their 638 valuable discussion.

639 Conflict of interest: The authors declare no conflict of interest.

\section{Figure Legends}




\section{Figure 1}

643 Multiple sequence alignment of ETMP1 orthologs in Entamoeba. Amino acid

644 sequences of orthologs in E. histolytica (EHI_175060), E. nutttalli (ENU1_040700), E.

645 dispar (EDI_139180), and E. moshkovskii (EMO_001640) were aligned using MAFFT

646 (67), and displayed using Jalview (68). The hydrophobic, positively charged, negatively

647 charged, polar, cysteine, glycine, proline, aromatic residues are indicated in blue, red,

648 magenta, green, pink, orange, yellow, and cyan respectively. Dashed black boxes show

649 predicted coiled coil domains by DeepCoil (64), while the dashed red box indicates the

650 predicted transmembrane region by our TMD prediction tool (12).

\section{$651 \quad$ Figure 2}

652 Expression and localization of HA-ETMP1 in E. histolytica trophozoites. (A)

653 Approximately $30 \mu \mathrm{g}$ protein from whole cell lysates of HA-ETMP1 and mock control

654 (pEhEx-HA) strains were separated by SDS-PAGE and subjected to anti-HA immunoblot 655 analysis. The $33 \mathrm{kDa}$ band corresponds to the predicted molecular mass of HA-ETMP1

656 (white arrowhead). (b) Immunofluorescence analysis of HA-ETMP1-expressing 657 trophozoites, double stained with anti-HA (green) and anti-APSK (red) respectively. Scale 658 bar $=10 \mu \mathrm{m}$. (C) Fractionation of HA-ETMP1 by discontinuous Percoll-gradient 659 ultracentrifugation. Homogenate of HA-ETMP1 was separated by density against a 660 Percoll gradient. Approximately $15 \mu \mathrm{L}$ of fractions collected from the first (1 to 22 ) and 661 second (A to V) ultracentrifugation steps were separated by SDS-PAGE followed by 662 immunoblot analysis with anti-HA and anti-Cpn60 antibodies respectively. (D) Anti-HA 663 and anti-Cpn60 immunoblot profiles of subcellular fractionation including alkaline 664 carbonate treated organelle-rich fractions of HA-ETMP1 and HA-MBOMP30 (mitosome 
665 membrane control) respectively. (E) Representative immunoelectron micrographs of 15

$666 \mathrm{~nm}$ anti-APSK gold-labeled mitosomes of HA-ETMP1, co-stained with $5 \mathrm{~nm}$ anti-HA gold.

667 Scale bar $=200 \mathrm{~nm}$.

668 Figure 3

669 Growth curve of HA-ETMP1 and mock-HA strain. Cell numbers of ETMP1 (black line)

670 and mock-HA strain (red line) cultivated in BI-S-33 medium containing 0, 10, and 20

$671 \mu \mathrm{g} / \mathrm{mL}$ G418 respectively, were plotted against time (h). Western blot analysis of whole

672 cell lysates of HA-ETMP1 and mock-HA grown in medium containing 0, 10, and $20 \mu \mathrm{g} / \mathrm{mL}$

673 G418, and harvested at various time points. Upper and lower panels show anti-HA and

674 anti-CPBF1 (loading control) immunoblots respectively.

\section{$675 \quad$ Figure 4}

676 Anti-HA beads immunoprecipitation (IP) of mock-HA and HA-ETMP1 strains. (A)

677 Western blot analysis using anti-HA antibody of the cell lysates and various IP fractions

678 of HA- mock-HA (left) and ETMP1 (right) respectively. A black arrowhead indicates the

679 position of HA-tagged ETMP1 (33 kDa). (B) Silver stained-SDS-PAGE gel of IP eluates

680 of mock-HA and HA-ETMP1 strains respectively. A black arrowhead points to a specific

$681 \sim 55 \mathrm{kDa}$ band unique to HA-ETMP1. (C) Enriched or exclusively detected proteins in the

$682 \sim 55 \mathrm{kDa}$ excised gel band from HA-ETMP1 IP eluate as compared to that of mock-HA

683 control IP eluate by LC-MS/MS sequencing analysis. MW stands for predicted molecular

684 weight. QV denotes quantitative values (normalized total spectra). Presence of the

685 detected proteins in the previously published mitosome proteome data (Mi-ichi et al.,

686 2009) was performed and the result listed in the last column (+ indicates presence, -

687 indicates absence). (D) Total cell lysates of mock-HA and ETMP1 respectively were 
separated by BN-PAGE, followed by anti-HA Western blot analysis. Black and red arrowheads respectively indicate the $\sim 180 \mathrm{kDa}$ and $\sim 90 \mathrm{kDa}$ complexes that contain HAETMP1.

HA-EHD1 expression in E. histolytica trophozoites. (A) Anti-HA immunoblot analysis

693 of approximately $30 \mu \mathrm{g}$ total cell lysates of mock-HA and HA-EHD1, respectively, show a $69461 \mathrm{kDa}$ band corresponding to HA-tagged EHD1. (B-C) Representative 695 immunofluorescence images of fixed HA-EHD1 expressing cells double-stained with anti696 HA (green) and anti-APSK (red) antibodies respectively. White arrow and arrowheads 697 indicate proximity and colocalization between anti-HA and anti-APSK signals respectively. 698 Scale bar $=10 \mu \mathrm{m}$. (D) Representative immunoelectron micrographs of HA-EHD1 699 trophozoites, double stained with $5 \mathrm{~nm}$ anti-HA gold and $15 \mathrm{~nm}$ anti-APSK gold. Scale 700 bar = $200 \mathrm{~nm}$. The letters "c", "e", and "m" indicate cytosol, endosomes, and mitosomes, 701 respectively. An arrowhead points to the structure where the membranes of the mitosome 702 and endosome are in close contact. (E) Percoll-gradient fractionation of HA-EHD1 703 followed by western blot analysis using anti-HA and ant-Cpn60 antibodies respectively.

704 Figure 6

705 Association of HA-EHD1 to E. histolytica membranes. (A) Colocalization analysis of 706 HA-EHD1 with various endosomal markers. Representative IFA images HA-EHD1 co707 stained with anti-HA (green) and anti-vacuolar protein sorting 26 (Vps26, red, upper 708 panel), anti-pyridine nucleotide transhydrogenase (PNT, red, middle panel), and anti709 Rab11B (red, bottom panel) respectively. (B) Immunoblot analysis of carbonate 710 fractionation assay of HA-EHD1 organelle-rich fraction using (from top to bottom panel) 
711 anti-HA, anti-CS1 (cytosolic protein control) anti-Sec13 (peripheral membrane protein

712 control), and anti-CPBF1 (membrane protein control) respectively. (C) Lipid overlay assay

713 of HA-EHD1 and HA-SNX1 (PI3P binding protein control) respectively. The membrane

714 strips contain 100 pmol of the following lipids per spot: lysophosphatidic acid (LPA),

715 lysophosphocholine (LPC), phosphatidylinositol (Ptdlns), phosphatidylinositol (3)-

716 phosphate (Ptdlns(3)P), phosphatidylinositol (4)-phosphate $\quad(P t d l n s(4) P)$,

717 phosphatidylinositol (5)-phosphate (Ptdlns(5)P), phosphatidylethanolamine (PE)

718 phosphatidylcholine (PC), sphingosine 1-phosphate (S1P), phosphatidylinositol $(3,4)$ -

719 bisphosphate (Ptdlns(3,4)P2), phosphatidylinositol $(3,5)$-bisphosphate (Ptdlns(3,5)P2),

720 phosphatidylinositol (4,5)-bisphosphate (Ptdlns(4,5)P2), phosphatidylinositol $(3,4,5)$ -

721 trisphosphate (Ptdlns(3,4,5)P3), phosphatidic acid (PA), phosphatidylserine (PS)

722 respectively.

\section{$723 \quad$ Figure 7}

724 Involvement of HA-EHD1 in multivesicular body formation. (A) Representative anti725 HA antibody and anti-APSK antiserum (upper panel) or anti-Vps26 antiserum (lower 726 panel) double-staining IFA images of trophozoites that expressed HA-EHD1 trophozoites

727 after $1 \mathrm{~h}, 3 \mathrm{~h}$, and $24 \mathrm{~h}$ of induction by tetracycline. Scale bar $=10 \mu \mathrm{m}$. (B) Representative 728 immunoelectron image of trophozoite expressing HA-EHD1 $24 \mathrm{~h}$ post-tetracycline 729 induction, stained with $15 \mathrm{~nm}$ gold anti-HA. The initials "C", "MVB", and "ILV" denote 730 cytosol, multivesicular body and intraluminal vesicle, respectively. Scale bar $=200 \mathrm{~nm}$.

\section{$731 \quad$ Figure 8}

732 Involvement of GFP-EHD1 in amoebic endocytosis. (A) Anti-GFP immunoblot analysis 733 of approximately $20 \mu \mathrm{g}$ total lysate of GFP-EHD1 expressing trophozoite. (B) Confocal 
734 microscopy images from movies of live trophozoites expressing GFP-EHD1 (left panel),

735 and GFP-EHD1 in medium supplemented with either RITC-dextran (middle panel) or

736 Alexa Fluor 568-transferrin (right panel) respectively. Scale bar $=10 \mu \mathrm{m}$.

737 Figure 9

738 Involvement of HA-EHD1 in amoebic phagocytosis and trogocytosis.

739 Representative IFA images of fixed anti-HA (green) and anti-Vps26 (red) double-stained

740 HA-EHD1 trophozoites 15, 30, and 60 minutes (top to bottom) after coincubation with

741 CellTracker Blue-stained Chinese hamster ovary $(\mathrm{CHO})$ cells.

742 Figure 10

743 Activity assay of purified recombinant His-EHD1. (A) Coomassie Brilliant Blue-stained

744 SDS-PAGE gel (left panel) and anti-His immunoblot (right panel) of purification fractions

745 of His-EHD1. (C) Determination of the specific activity of His-EHD1 using ATP as

746 substrate at various concentrations.

747

748 Supplemental Materials

749 Supplementary Movie S1

750 Multiple z-section images of fixed HA-EHD1, $24 \mathrm{~h}$ after tetracycline induction. Green and

751 red signals indicate anti-HA and anti-APSK antibodies respectively. Scale bar $=5 \mu \mathrm{m}$.

\section{Supplementary Movie S2}

753 Live imaging of GFP-EHD1 after 24 of tetracycline induction. Scale bar $=5 \mu \mathrm{m}$.

\section{Supplementary Movie S3}


755 Live imaging of GFP-EHD1 trophozoites chased a few minutes after addition of RITC-

756 dextran. Note the recruitment of GFP-EHD1 in newly closed endosomes. Scale bar $=5$

$757 \mu \mathrm{m}$.

\section{Supplementary Movie S4}

759 Live imaging of GFP-EHD1 trophozoites chased a few minutes after addition of Alexa

760 Fluor 568-transferrin. Note the recruitment of GFP-EHD1 in newly closed endosomes.

\section{Supplementary Movie S5}

762 Live imaging of GFP-EHD1 trophozoites chased a few minutes after addition of Alexa

763 Fluor 568-transferrin. Note the accumulation of GFP-EHD1 in the plasma membrane

764 where aggregated transferrin is located. Scale bar $=5 \mu \mathrm{m}$.

\section{Supplementary Movie S6}

766 Multiple z-section images of fixed GFP-EHD1, (60 min after co-incubation with

767 CellTracker blue-stained Chinese hamster ovary cells. Scale bar $=5 \mu \mathrm{m}$.

\section{Supplementary Movie S7}

769 Live imaging of GFP-EHD1 trophozoites chased a few minutes after co-incubation with

770 CellTracker blue-stained Chinese hamster ovary cells. Scale bar $=5 \mu \mathrm{m}$.

\section{Supplementary Figure S1}

772 Anti-HA immunoprecipitation of HA-EHD1 and mock-HA control. (A) Western blot

773 analysis of various IP fractions probed using anti-HA antibody (B) Silver-stained SDS-

774 PAGE gel showing separated protein bands from eluted IP samples. Black boxes

775 indicated regions excised and submitted for subsequent protein sequencing analysis.

\section{Supplementary Table S1}


777 (A) Exclusively detected proteins in the $\sim 180 \mathrm{kDa}$ excised blue-native PAGE gel band

778 from HA-ETMP1 as compared to that of mock-HA control by LC-MS/MS sequencing

779 analysis. MW indicates predicted molecular weight, while QV denotes quantitative values

780 (normalized total spectra). (B) Detected proteins in the $\sim 180 \mathrm{kDa}$ excised blue-native

781 PAGE gel band enriched in HA-ETMP1 as compared to that of mock-HA control by LC-

782 MS/MS sequencing analysis (Qv HA/ETMp1/mock-HA $\geq 2.0$.) (C) Exclusively detected

783 proteins in the $\sim 90 \mathrm{kDa}$ excised blue-native PAGE gel band enriched in HA-ETMP1 as

784 compared to that of mock-HA control by LC-MS/MS sequencing analysis.

\section{Supplementary Table S2}

786 (A) Proteins identified in the 55-58 kDa excised gel band of HA-EHD1 and mock-HA IP

787 eluate samples respectively, by LC-MS/MS analysis. MW indicates predicted molecular

788 weight, while QV denotes quantitative values (normalized total spectra). (B) Proteins

789 identified in the 30-33 kDa excised gel band of HA-EHD1 and mock-HA IP respectively,

790 by LC-MS/MS analysis. MW indicates predicted molecular weight, while QV denotes

791 quantitative values (normalized total spectra).

792

793

\section{References}

795 1. Jain A, Holthuis JCM. 2017. Membrane contact sites, ancient and central hubs of

796 cellular lipid logistics. Biochim Biophys Acta - Mol Cell Res 1864:1450-1458.

797 2. Helle SCJ, Kanfer G, Kolar K, Lang A, Michel AH, Kornmann B. 2013.

798 Organization and function of membrane contact sites. Biochim Biophys Acta - Mol

$799 \quad$ Cell Res 1833:2526-2541. 
800 3. Scorrano L, De Matteis MA, Emr S, Giordano F, Hajnóczky G, Kornmann B,

801 Lackner LL, Levine TP, Pellegrini L, Reinisch K, Rizzuto R, Simmen T, Stenmark

$802 \quad H$, Ungermann C, Schuldiner M. 2019. Coming together to define membrane

$803 \quad$ contact sites. Nat Commun 10:1-11.

804 4. Hoyer MJ, Chitwood PJ, Ebmeier CC, Striepen JF, Qi RZ, Old WM, Voeltz GK.

805 2018. A novel class of ER membrane proteins regulates ER-associated

$806 \quad$ endosome fission. Cell 175:254-265.

807 5. Wong YC, Ysselstein D, Krainc D. 2018. Mitochondria-lysosome contacts regulate 808 mitochondrial fission via RAB7 GTP hydrolysis. Nature 554:382-386.

809 6. Stanley SL. 2003. Amoebiasis. Lancet 361:1025-1034.

810 7. Santos HJ, Makiuchi T, Nozaki T. 2018. Reinventing an Organelle: The Reduced 811 Mitochondrion in Parasitic Protists. Trends Parasitol 1-18.

812 8. Mi-ichi F, Miyamoto T, Takao S, Jeelani G, Hashimoto T, Hara H, Nozaki T, 813 Yoshida H. 2015. Entamoeba mitosomes play an important role in encystation by 814 association with cholesteryl sulfate synthesis. Proc Natl Acad Sci 112:E2884$815 \quad$ E2890.

8169 9. Teixeira JE, Huston CD. 2008. Evidence of a continuous endoplasmic reticulum in 817 the protozoan parasite Entamoeba histolytica 7:1222-1226.

818 10. Perdomo D, Aït-Ammar N, Syan S, Sachse M, Jhingan GD, Guillén N. 2015.

819 Cellular and proteomics analysis of the endomembrane system from the 820 unicellular Entamoeba histolytica. J Proteomics 112:125-140.

821 11. Santos HJ, Imai K, Makiuchi T, Tomii K, Horton P, Nozawa A, Okada K, Tozawa 822 Y, Nozaki T. 2019. Novel lineage-specific transmembrane $\beta$-barrel proteins in the 
endoplasmic reticulum of Entamoeba histolytica. FEBS J 286:3416-3432.

824 12. Santos HJ, Imai K, Hanadate Y, Fukasawa Y, Oda T, Mi-ichi F, Nozaki T. 2016. Entamoeba histolytica. Mol Biochem Parasitol 209:10-17.

827 13. Santos HJ, Hanadate Y, Imai K, Nozaki T. 2019. An Entamoeba-Specific

Mitosomal Membrane Protein with Potential Association to the Golgi Apparatus.

830 14. Santos HJ, Nozaki T. 2021. Interorganellar communication and membrane contact sites in protozoan parasites. Parasitol Int 83:102372.

832 15. Saito-Nakano Y, Loftus BJ, Hall N, Nozaki T. 2005. The diversity of Rab GTPases in Entamoeba histolytica. Exp Parasitol 110:244-252.

834 16. Lopez-Reyes I, García-Rivera G, Bauelos C, Herranz S, Vincent O, Lpez-

Camarillo C, Marchat LA, Orozco E. 2010. Detection of the endosomal sorting complex required for transport in entamoeba histolytica and characterization of the EhVps4 protein. J Biomed Biotechnol 2010.

838 17. Avalos-Padilla Y, Knorr RL, Javier-Reyna R, García-Rivera G, Lipowsky R, Dimova R, Orozco E. 2018. The Conserved ESCRT-III Machinery Participates in the Phagocytosis of Entamoeba histolytica. Front Cell Infect Microbiol 8:1-17.

841 18. Mi-ichi F, Yousuf MA, Nakada-Tsukui K, Nozaki T. 2009. Mitosomes in Entamoeba histolytica contain a sulfate activation pathway. Proc Natl Acad Sci 106:21731-21736. is a novel Rab7 effector that is involved in the transport of the virulence factor 
cysteine protease in the enteric protozoan parasite Entamoeba histolytica. Mol Biol Cell2005/08/27. 16:5294-5303.

20. Watanabe N, Nakada-Tsukui K, Nozaki T. 2020. Two isotypes of phosphatidylinositol 3-phosphate-binding sorting nexins play distinct roles in trogocytosis in Entamoeba histolytica. Cell Microbiol 22:1-16.

21. Yousuf MA, Mi-Ichi F, Nakada-Tsukui K, Nozaki T. 2010. Localization and targeting of an unusual pyridine nucleotide transhydrogenase in Entamoeba histolytica. Eukaryot Cell 9:926-933.

22. Mitra BN, Saito-Nakano Y, Nakada-Tsukui K, Sato D, Nozaki T. 2007. Rab11B small GTPase regulates secretion of cysteine proteases in the enteric protozoan parasite Entamoeba histolytica. Cell Microbiol 9:2112-2125.

23. Makiuchi T, Mi-ichi F, Nakada-Tsukui K, Nozaki T. 2013. Novel TPR-containing subunit of TOM complex functions as cytosolic receptor for Entamoeba mitosomal

24. Santos HJ, Imai K, Makiuchi T, Tomii K, Horton P, Nozawa A, Ibrahim M, Tozawa Y, Nozaki T. 2015. A novel mitosomal $\beta$-barrel outer membrane protein in Entamoeba. Sci Rep 5:1-10.

25. Galperin E, Benjamin S, Rapaport DD, Rotem-Yehudar R, Tolchinsky S, Horowitz M. 2002. EHD3: A protein that resides in recycling tubular and vesicular membrane structures and interacts with EHD1. Traffic 3:575-589.

26. George M, Ying GG, Rainey MA, Solomon A, Parikh PT, Gao Q, Band V, Band H. 2007. Shared as well as distinct roles of EHD proteins revealed by biochemical and functional comparisons in mammalian cells and C. elegans. BMC Cell Biol 
870 27. Confalonieri S, Di Fiore PP. 2002. The Eps15 homology (EH) domain. FEBS Lett 513:24-29.

872 28. Yeow I, Howard G, Chadwick J, Mendoza-Topaz C, Hansen CG, Nichols BJ, Shvets E. 2017. EHD Proteins Cooperate to Generate Caveolar Clusters and to Maintain Caveolae during Repeated Mechanical Stress. Curr Biol 27:29512962.e5.

876

29. Melo AA, Hegde BG, Shah C, Larsson E, Isas JM, Kunz S, Lundmark R, Langen R, Daumke O. 2017. Structural insights into the activation mechanism of dynaminlike EHD ATPases. Proc Natl Acad Sci U S A 114:5629-5634.

30. Naslavsky N, Rahajeng J, Rapaport D, Horowitz M, Caplan S. 2007. EHD1 regulates cholesterol homeostasis and lipid droplet storage. Biochem Biophys Res Commun 357:792-799.

31. Bar M, Aharon M, Benjamin S, Rotblat B, Horowitz M, Avni A. 2008. AtEHDs, novel Arabidopsis EH-domain-containing proteins involved in endocytosis. Plant J 55:1025-1038.

32. Lin SX, Grant B, Hirsh D, Maxfield FR. 2001. Rme-1 regulates the distribution and function of the endocytic recycling compartment in mammalian cells. Nat Cell Biol

33. Thakur V, Asad M, Jain S, Hossain ME, Gupta A, Kaur I, Rathore S, Ali S, Khan 
1853:2856-2869.

893 34. Gueho A, Bosmani C, Gopaldass N, Molle V, Soldati T, Letourneur F. 2016. maturation. J Cell Sci 129:2354-2367.

35. Daumke O, Lundmark R, Vallis Y, Martens S, Butler PJG, McMahon HT. 2007.

Architectural and mechanistic insights into an EHD ATPase involved in membrane remodelling. Nature 449:923-927.

36. Sharma M, Giridharan SSP, Rahajeng J, Naslavsky N, Aplan S. 2009. MICAL-L1

37. Pant S, Sharma M, Patel K, Caplan S, Carr CM, Grant BD. 2009. AMPHBiol 11:1399-1410.

38. Lee DW, Zhao X, Scarselletta S, Schweinsberg PJ, Eisenberg E, Grant BD, association of RME-1 family proteins. J Biol Chem 280:17213-17220.

39. Stoeber M, Stoeck IK, HéCurrency Signnni C, Bleck CKE, Balistreri G, Helenius

911 40. Deo R, Kushwah MS, Kamerkar SC, Kadam NY, Dar S, Babu K, Srivastava A, to endocytic recycling. Nat Commun 9.

914 41. Nakada-Tsukui K, Watanabe N, Maehama T, Nozaki T. 2019. 
Phosphatidylinositol Kinases and Phosphatases in Entamoeba histolytica. Front Cell Infect Microbiol 9:1-36.

917 42. Koushik AB, Powell RR, Temesvari LA. 2013. Localization of phosphatidylinositol 918 4,5-bisphosphate to lipid rafts and uroids in the human protozoan parasite entamoeba histolytica. Infect Immun 81:2145-2155.

920 43. Swanson JA. 2014. Phosphoinositides and engulfment. Cell Microbiol 16:14731483.

922 44. Wallroth A, Haucke V. 2018. Phosphoinositide conversion in endocytosis and the endolysosomal system. J Biol Chem 293:1526-1535.

924 45. Odorizzi G, Babst M, Emr SD. 1998. Fab1p Ptdlns(3)P 5-kinase function essential for protein sorting in the multivesicular body. Cell 95:847-858.

46. Das K, Watanabe N, Nozaki T. 2021. Two StAR-related lipid transfer proteins play specific roles in endocytosis, exocytosis, and motility in the parasitic protist Entamoeba histolytica. PLoS Pathog 17:1-27.

47. Das A, Nag S, Mason AB, Barroso MM. 2016. Endosome-mitochondria interactions are modulated by iron release from transferrin. J Cell Biol 214:831845.

932 48. Farmer T, Reinecke JB, Xie S, Bahl K, Naslavsky N, Caplan S. 2017. Control of mitochondrial homeostasis by endocytic regulatory proteins. J Cell Sci 130:2359_ 2370.

935 49. Wexler-Cohen Y, Stevens GC, Barnoy E, Van Der Bliek AM, Johnson PJ. 2014. A 
938 50. Makiuchi T, Santos HJ, Tachibana H, Nozaki T. 2017. Hetero-oligomer of dynamin-related proteins participates in the fission of highly divergent mitochondria from Entamoeba histolytica. Sci Rep 7:1-13.

941 51. Santos HJ, Makiuchi T, Nozaki T. 2018. Reinventing an Organelle: The Reduced Mitochondrion in Parasitic Protists. Trends Parasitol 34:1038-1055.

943 52. Soubannier V, McLelland GL, Zunino R, Braschi E, Rippstein P, Fon EA, McBride HM. 2012. A vesicular transport pathway shuttles cargo from mitochondria to lysosomes. Curr Biol 22:135-141.

53. Li Z, Schulze RJ, Weller SG, Krueger EW, Schott MB, Zhang X, Casey CA, Liu J, Stöckli J, James DE, McNiven MA. 2016. A novel rab10-EHBP1-EHD2 complex essential for the autophagic engulfment of lipid droplets. Sci Adv 2.

54. Diamond LS, Harlow DR, Cunnick CC. 1978. A new medium for the axenic cultivation of Entamoeba histolytica and other Entamoeba. Trans R Soc Trop Med

55. Bracha R, Nuchamowitz Y, Anbar M, Mirelman D. 2006. Transcriptional silencing of multiple genes in trophozoites of Entamoeba histolytica. PLoS Pathog2006/05/31. 2:e48.

955 56. Nakada-Tsukui K, Okada H, Mitra BN, Nozaki T. 2009. Phosphatidylinositolphosphates mediate cytoskeletal reorganization during phagocytosis via a unique modular protein consisting of RhoGEF/DH and FYVE domains in the parasitic protozoon Entamoeba histolytica. Cell Microbiol 11:1471-1491. mitosomes plays an important role in the proliferation of Entamoeba histolytica. 
PLoS Negl Trop Dis 5:e1263.

962 58. Nozaki T, Asai T, Sanchez LB, Kobayashi S, Nakazawa M, Takeuchi T. 1999.

59. Chiba Y, Kamikawa R, Nakada-Tsukui K, Saito-Nakano Y, Nozaki T. 2015. and Bacteria. J Biochem 290:23960-23970.

969

970

971

972

973

974

975

976

977

978

979

980

981

982

983

60. Tomii K, Santos HJ, Nozaki T. 2019. Genome-wide analysis of known and potential tetraspanins in Entamoeba histolytica. Genes (Basel) 10:1-19.

61. Nozaki T, Asai T, Kobayashi S, Ikegami F, Noji M, Saito K, Takeuchi T. 1998. Molecular cloning and characterization of the genes encoding two isoforms of cysteine synthase in the enteric protozoan parasite Entamoeba histolytica. Mol Biochem Parasitol 97:33-44.

62. Nakada-Tsukui K, Tsuboi K, Furukawa A, Yamada Y, Nozaki T. 2012. A novel class of cysteine protease receptors that mediate lysosomal transport. Cell Microbiol 14:1299-1317.

63. Aurrecoechea C, Barreto A, Brestelli J, Brunk BP, Caler E V., Fischer S, Gajria B, Gao X, Gingle A, Grant G, Harb OS, Heiges M, lodice J, Kissinger JC, Kraemer ET, Li W, Nayak V, Pennington C, Pinney DF, Pitts B, Roos DS, Srinivasamoorthy G, Stoeckert CJ, Treatman C, Wang H. 2011. AmoebaDB and MicrosporidiaDB: Functional genomic resources for Amoebozoa and Microsporidia species. Nucleic Acids Res 39:1-8. 
984 64. Ludwiczak J, Winski A, Szczepaniak K, Alva V, Dunin-Horkawicz S. 2019.

985 DeepCoil - A fast and accurate prediction of coiled-coil domains in protein sequences. Bioinformatics 35:2790-2795.

987 65. Nurkanto A, Jeelani G, Santos HJ, Rahmawati Y, Mori M, Nakamura Y, Goto K,

Saikawa Y, Annoura T, Tozawa Y, Sakura T, Inaoka DK, Shiomi K, Nozaki T.

2021. Characterization of Plasmodium falciparum Pantothenate Kinase and

Identification of Its Inhibitors From Natural Products. Front Cell Infect Microbiol

$11: 1-10$

992 66. Kumagai K, Kojima H, Okabe T, Nagano T. 2014. Development of a highly sensitive, high-throughput assay for glycosyltransferases using enzyme-coupled fluorescence detection. Anal Biochem 447:146-155.

67. Katoh K, Standley DM. 2013. MAFFT multiple sequence alignment software version 7: Improvements in performance and usability. Mol Biol Evol 30:772-780.

997 68. Waterhouse AM, Procter JB, Martin DMA, Clamp M, Barton GJ. 2009. Jalview Version 2-a multiple sequence alignment editor and analysis workbench. 
10

20

30

40

EHI_175060 1 MEQ I TEEA I NQS I I EHD I NKT I QLCEAYLKN I V VPGKQSKEVENVF ENU1_040700

EDI 139180 EMO_001640 1 MEQ I TEEA I NQS I I END I NKT I QLCELYLKN I VVTGKQSKEVENVF 1 MEQ I TEEA I NQS I I EKDTNKT I QLCELYLKN I VVPGKQSKEVENVF 1 MED I TVDA I NQSV I ENDNSKT I QLCELYLKN I SSPGKQSKEVEDVF

$50 \quad 60070 \quad 8009$

EHI_175060 47 IHYLHAMSNTGEYEK I KKMFDQLYSK I SDASGNVA I MY I NVLVLND ENU1_040700 47 I HYLHAMSNTGEYEK I KKMF DQLYSK I SDASSNVA I MY I NVLVLND

EDI 139180 EMO_001640 47 I HYLHA I SNTDEYEK I KKMFDQLYSK I SDASDNVA I MY I NALVLND 47 VHY I HAMSNRDGFEK I KEMFEKLYSN I SDSSCDVAVMY I NVL I LND

100 110 120 130

EHI_175060 ENU1_040700

EDI-139180 EMO_001640

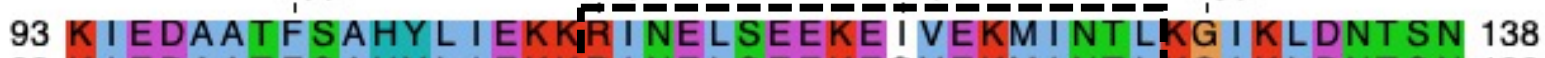
93 K I EDAATFSAHYL I EKKIR I NELSEEKESVEKM I NT LKG I KLDNT SN 138 93 K I EDAATFSAHYL I EKKIR I NELSEEKE I VEKM I NT LKE I KLDNTSN 138 93 K I EDAA I FSAHYLVQKQKLLNELENENET I EKL I CNLAA I KMDNSGY 138 140 160 170 180

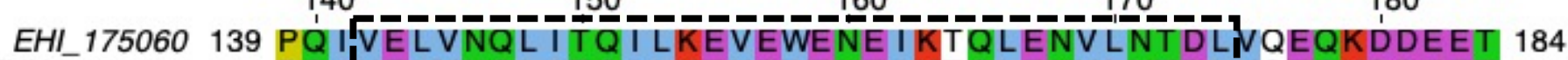
ENU1_040700 139 PQ IJVELVNQL I TQ I LKEVDWENE I KTQLENVLNTD LNQEQKDDEET 184

EDI_139180 $139 \mathrm{PQ}$ IIVE LVNQL I TEMLKEVDWENE I K I QLENV LNADLNPEQKDDDET 184 EMO_001640 139 SQ IIVELVDKLVNEALKEVEWDNE I K IQLLEN I LNSQL_. - - - NSTKS 179 190 200 210 220

EHI_175060 185 EEEEEE - DLGKYLQVSQVTF L N I WT RKLWRSACSLLDY I KG I MRKP 229 ENU1_040700 185 EEVEEV I DKNNY-QV SQVTF LN I WT RK LWRSACS LLDY I KG I MRKP 229

EDI_139180 185 EEEEVT - - E ENLQT TQTTF LNNCTRELWRS ACSLLDY I KG I MRKP 227 EMO_001640 180 ETDEVVLD-EQNLQVAN I SVFSEWSRKLWKSACS L I DY I KG I MRKP 224 
Figure 2

A

(kDa)

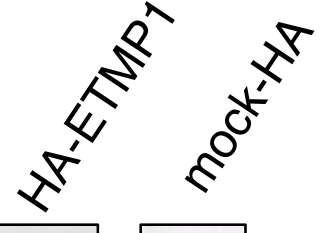

HA-ETMP1

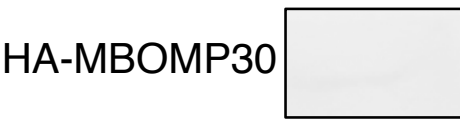

HA-ETMP1
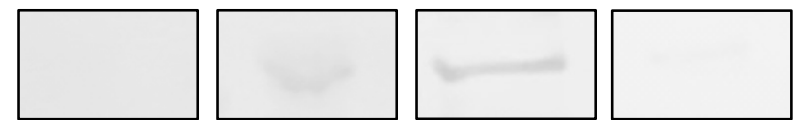

anti-Cpn60

E
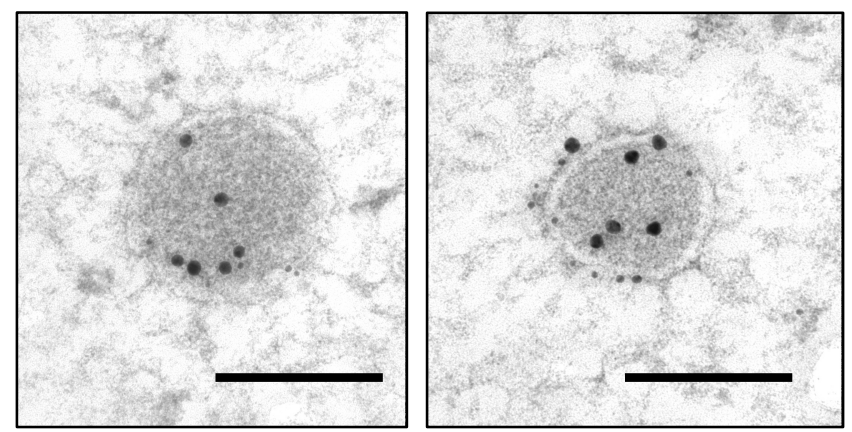

B
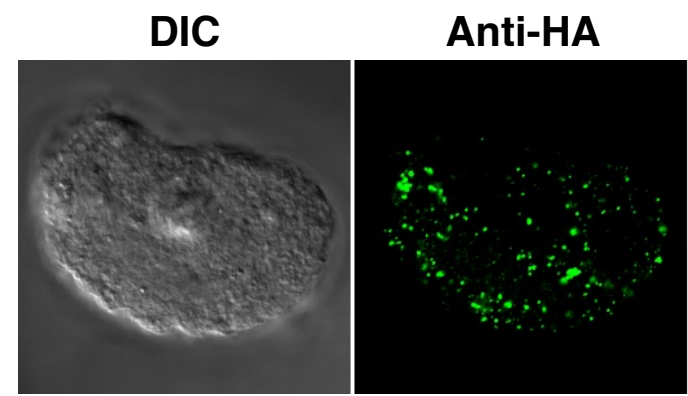

Anti-APSK

Merge
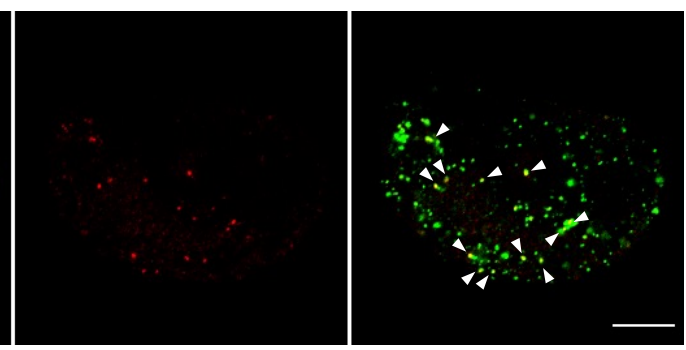

C First Percoll-gradient fractions

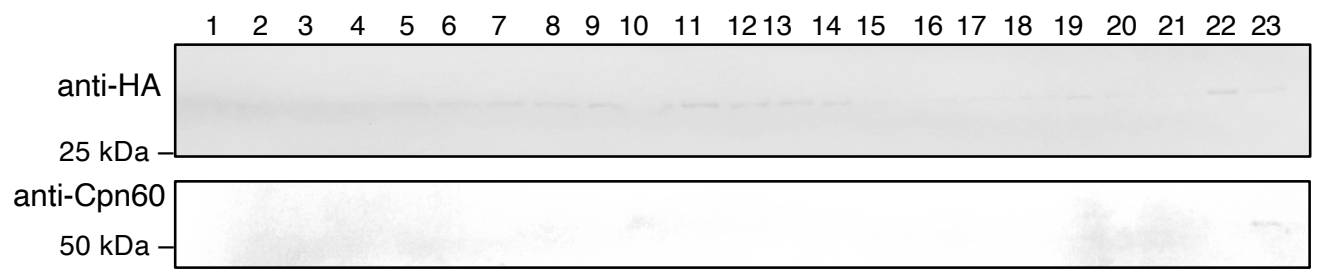

Second Percoll-gradient fractions

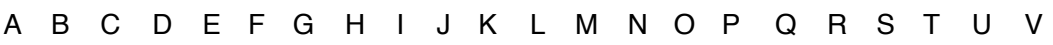

anti-HA

$25 \mathrm{kDa}$

anti-Cpn60

$50 \mathrm{kDa}$ 
A

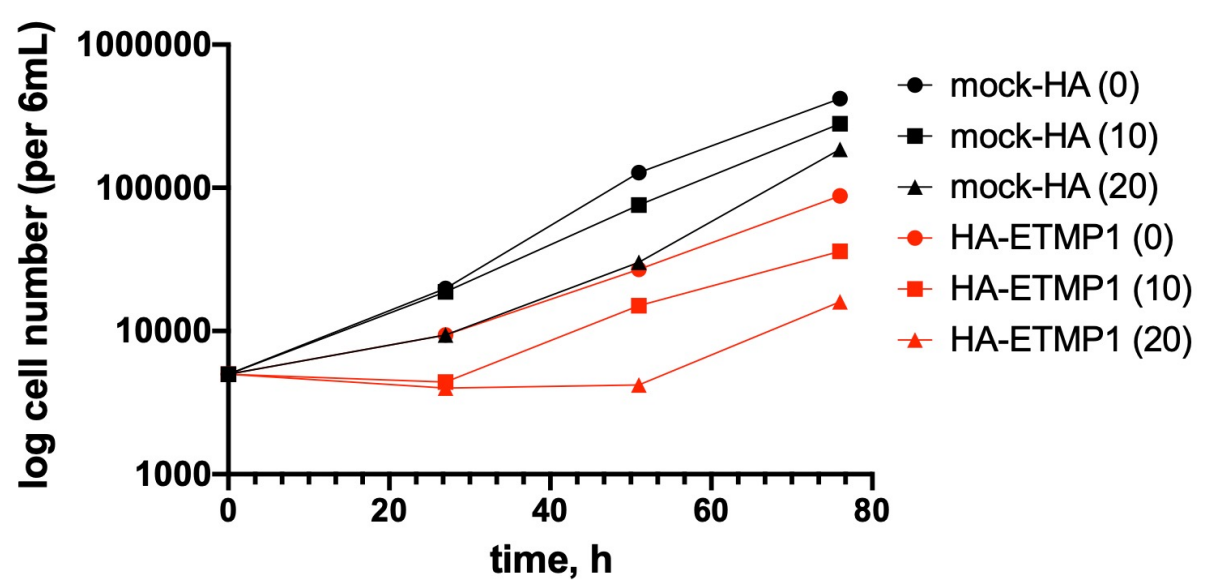

B

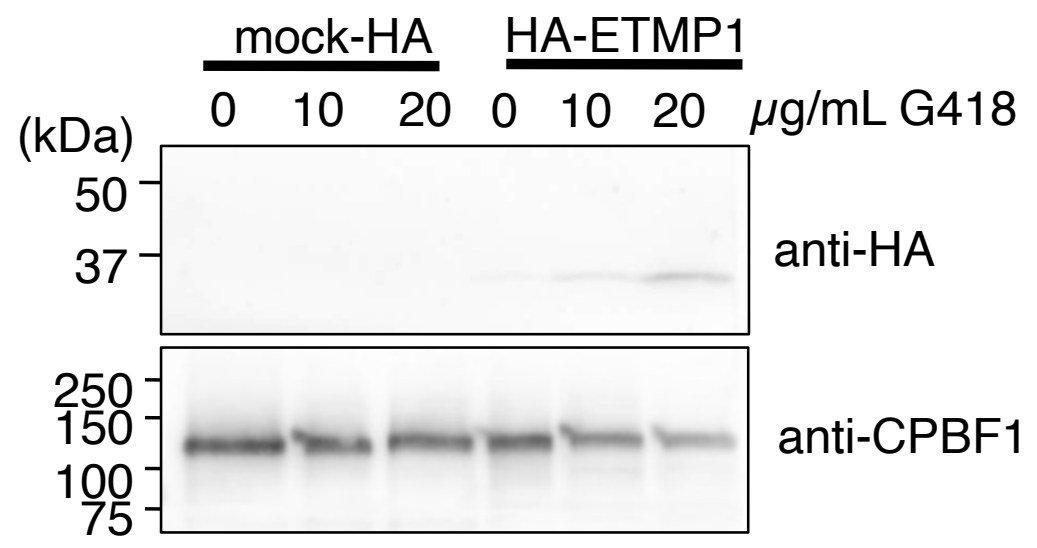


Figure 4

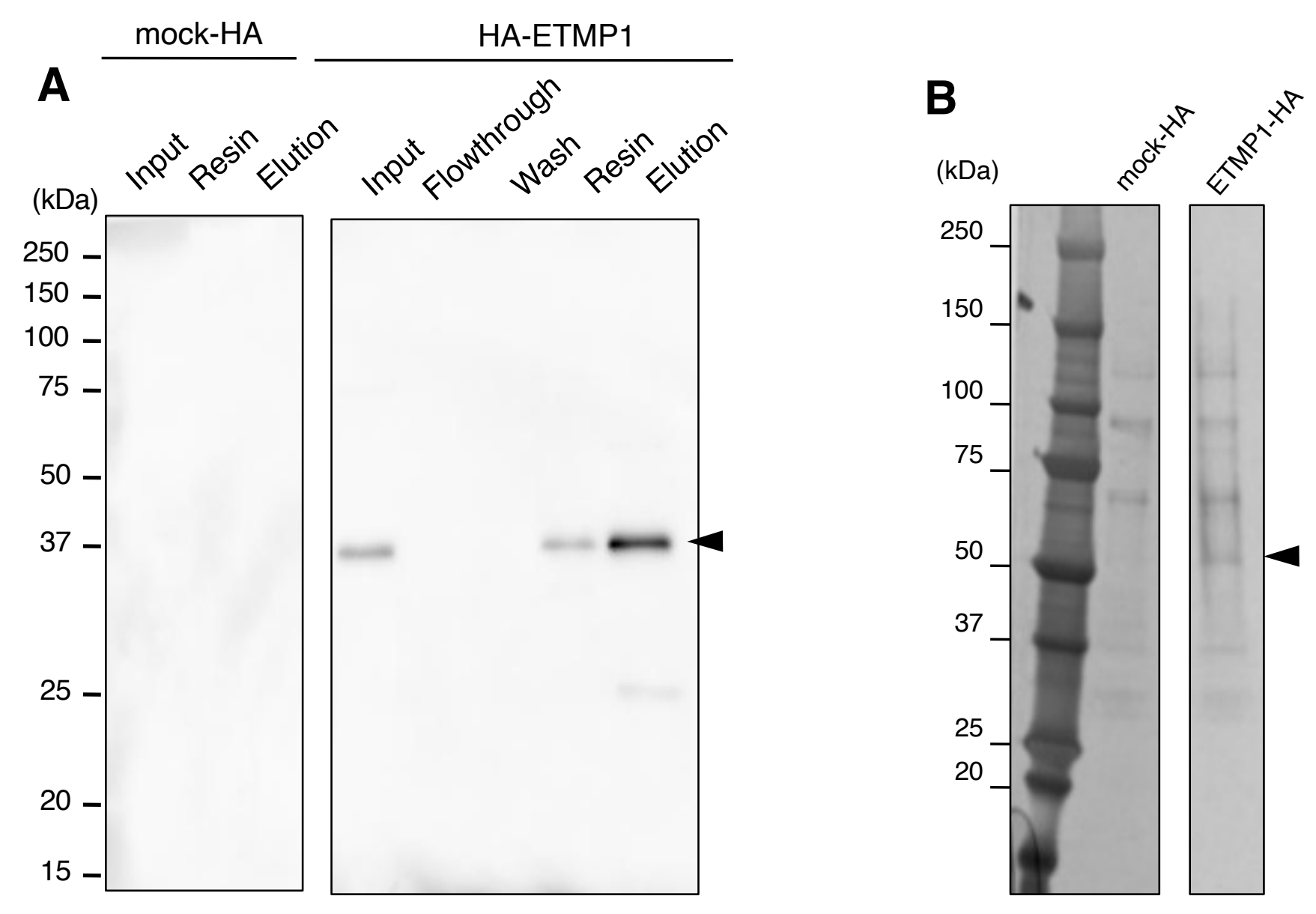

C

\begin{tabular}{cccccc}
\hline Protein Name & $\begin{array}{c}\text { Accession } \\
\text { Number }\end{array}$ & $\begin{array}{c}\text { MW } \\
\text { (kDa) }\end{array}$ & $\begin{array}{c}\text { Qv } \\
\text { HA- } \\
\text { ETMP1 }\end{array}$ & $\begin{array}{c}\text { Qv } \\
\text { mock- } \\
\text { HA }\end{array}$ & $\begin{array}{c}\text { Mitosome } \\
\text { proteome }\end{array}$ \\
\hline $\begin{array}{c}\text { L-myo-inositol-1- } \\
\text { phosphate synthase }\end{array}$ & EHI_070720 & 57 & 9 & 2 & + \\
\hline $\begin{array}{c}\text { Vacuolar protein } \\
\text { sorting-associated } \\
\text { protein 45 }\end{array}$ & EHI_154290 & 60 & 4 & 0 & - \\
\hline $\begin{array}{c}\text { EH-domain containing } \\
\text { protein (EHD1) }\end{array}$ & EHI_105270 & 58 & 2 & 0 & + \\
\hline $\begin{array}{c}\text { EH-domain containing } \\
\text { protein (EHD2) }\end{array}$ & EHI_152680 & 58 & 2 & 0 & + \\
\hline
\end{tabular}
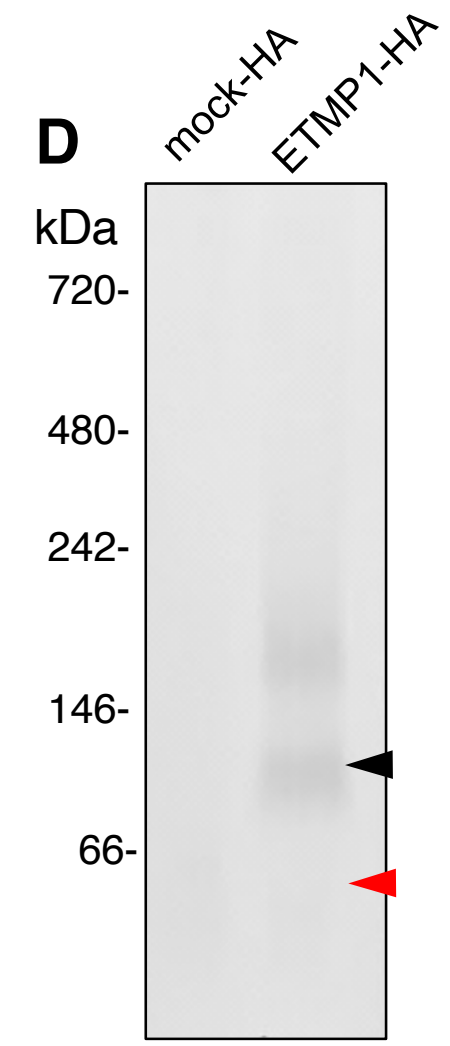
Figure 5

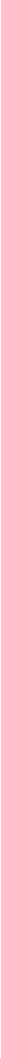

First Percoll-gradient fractions

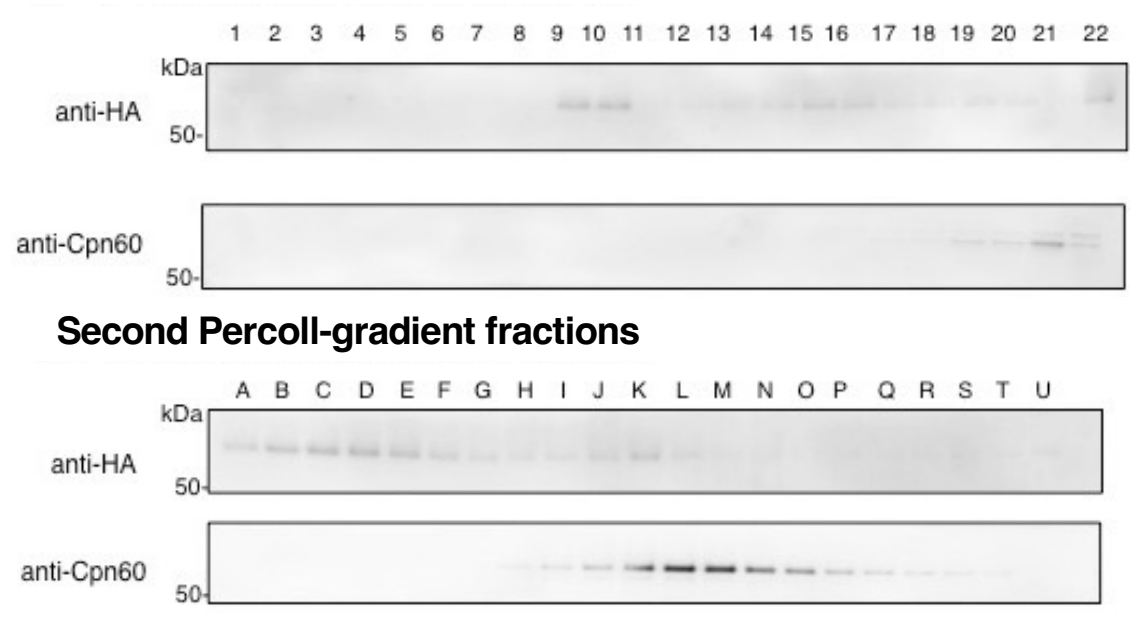



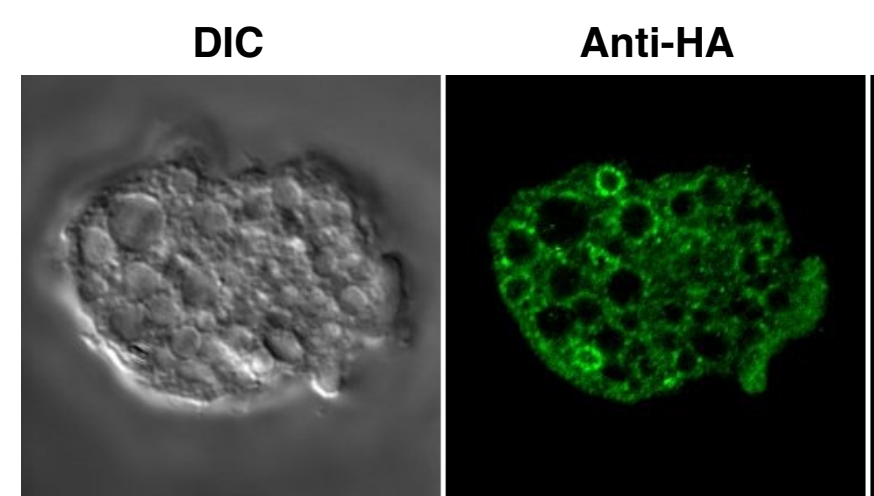

Anti-HA

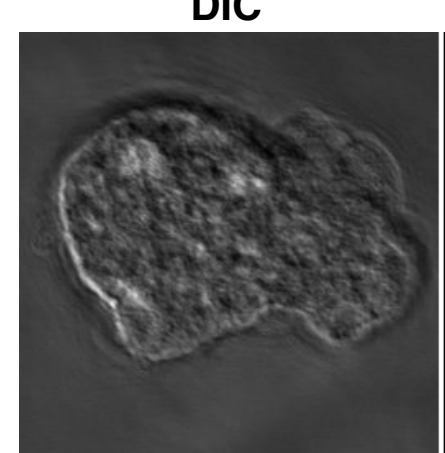

DIC

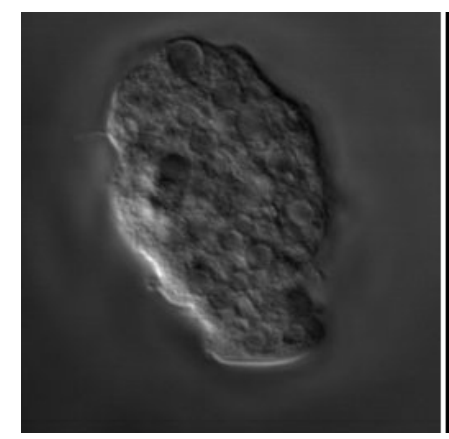

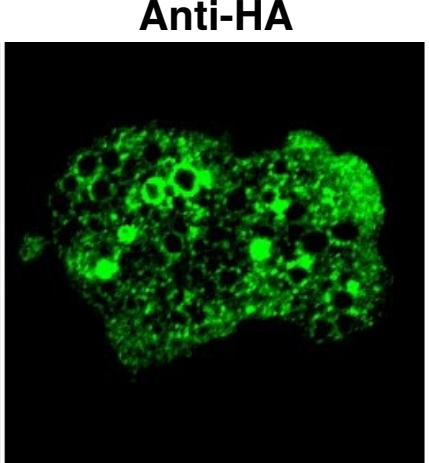

Anti-HA

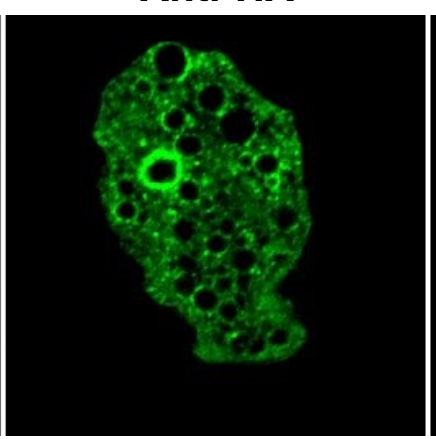

Anti-Vps26

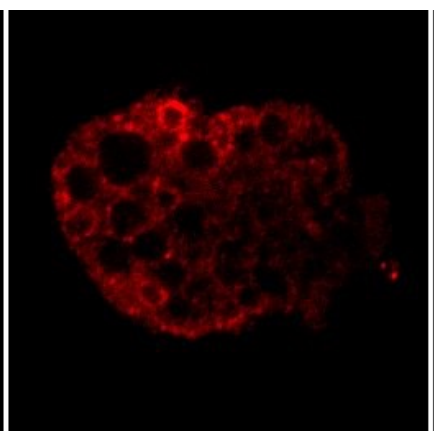

Anti-PNT

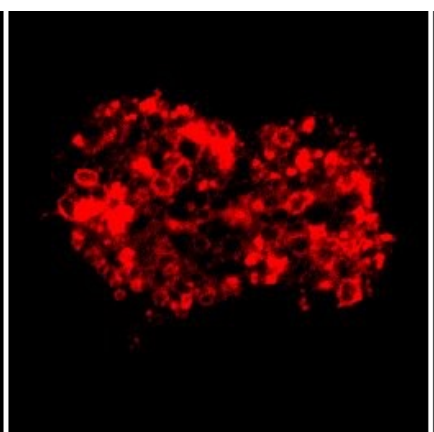

Anti-Rab11B

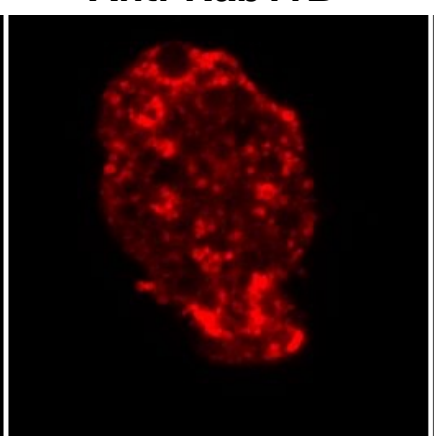

Merge

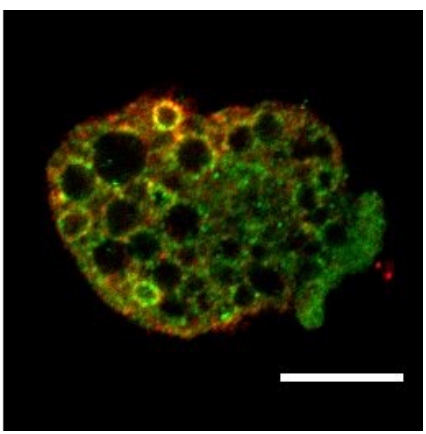

Merge

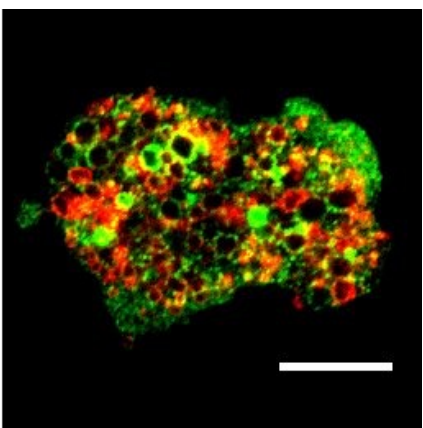

Merge

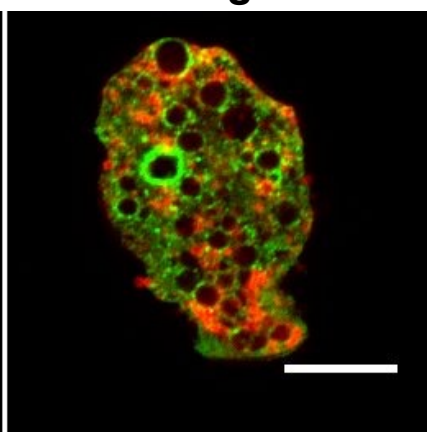

B

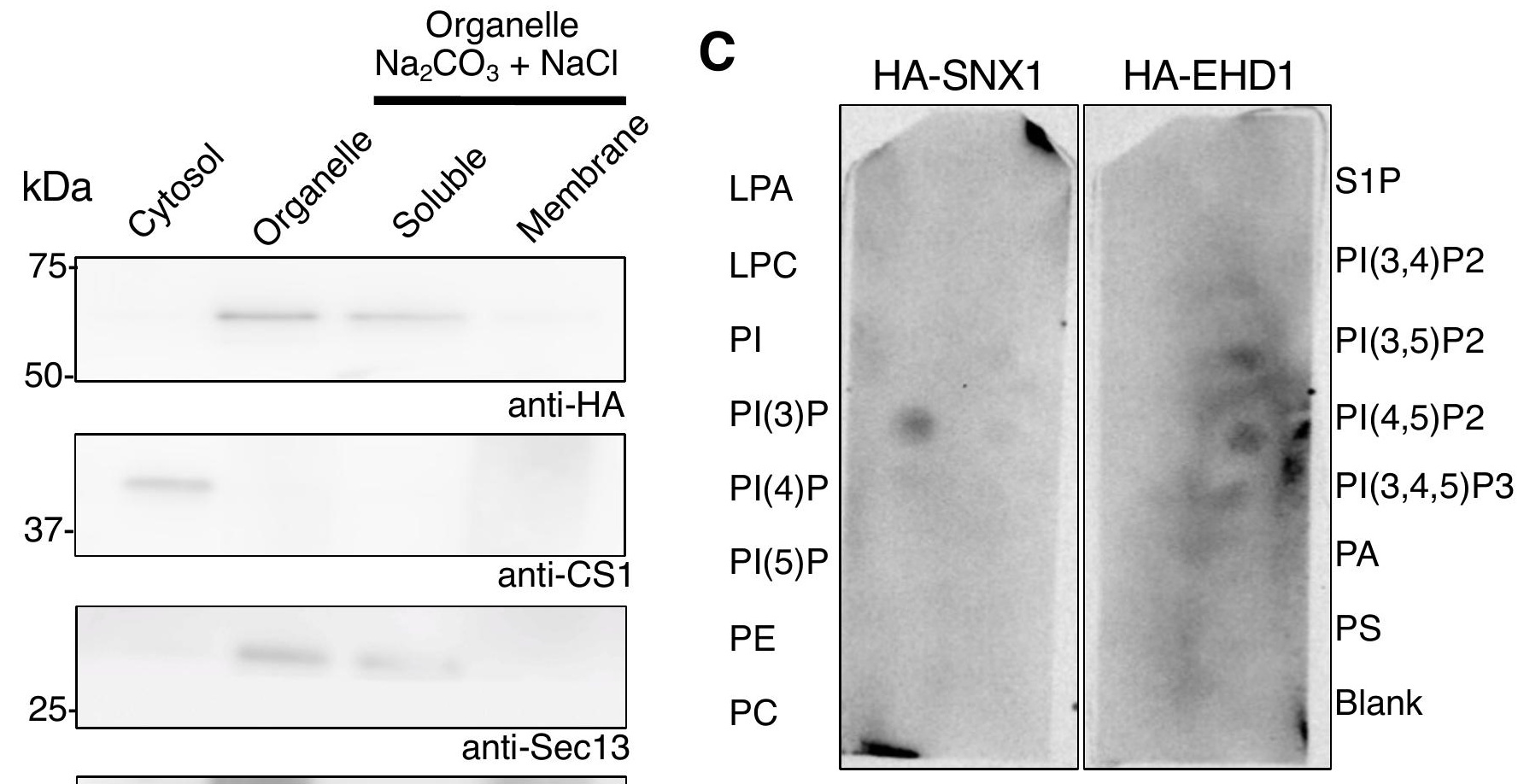


Figure 7

A

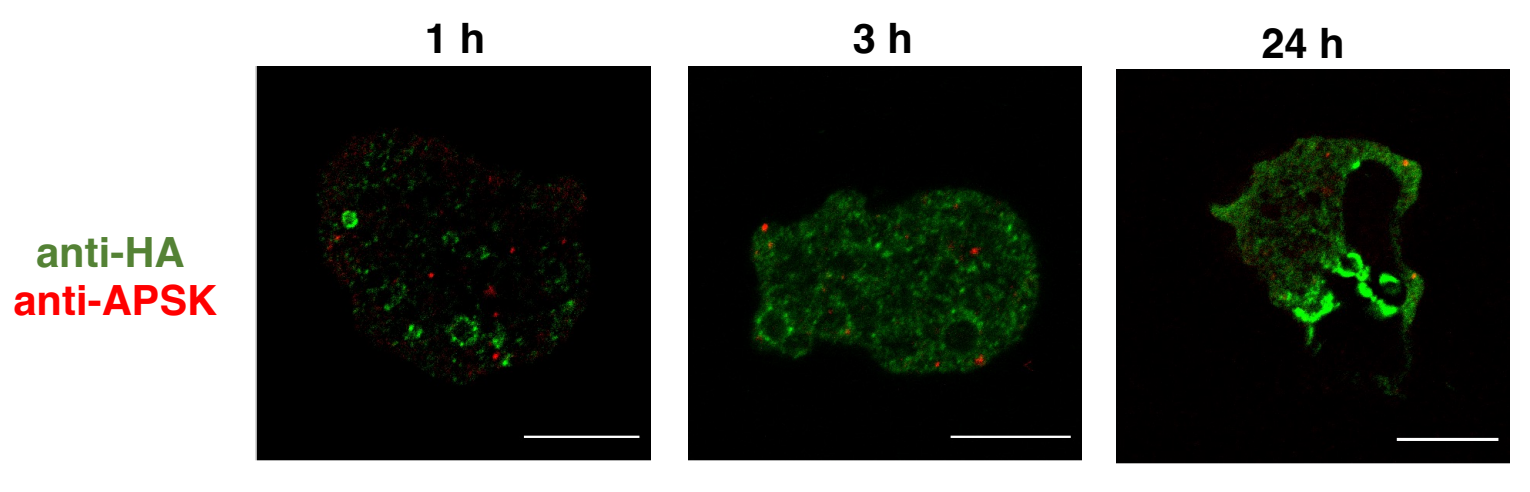

B
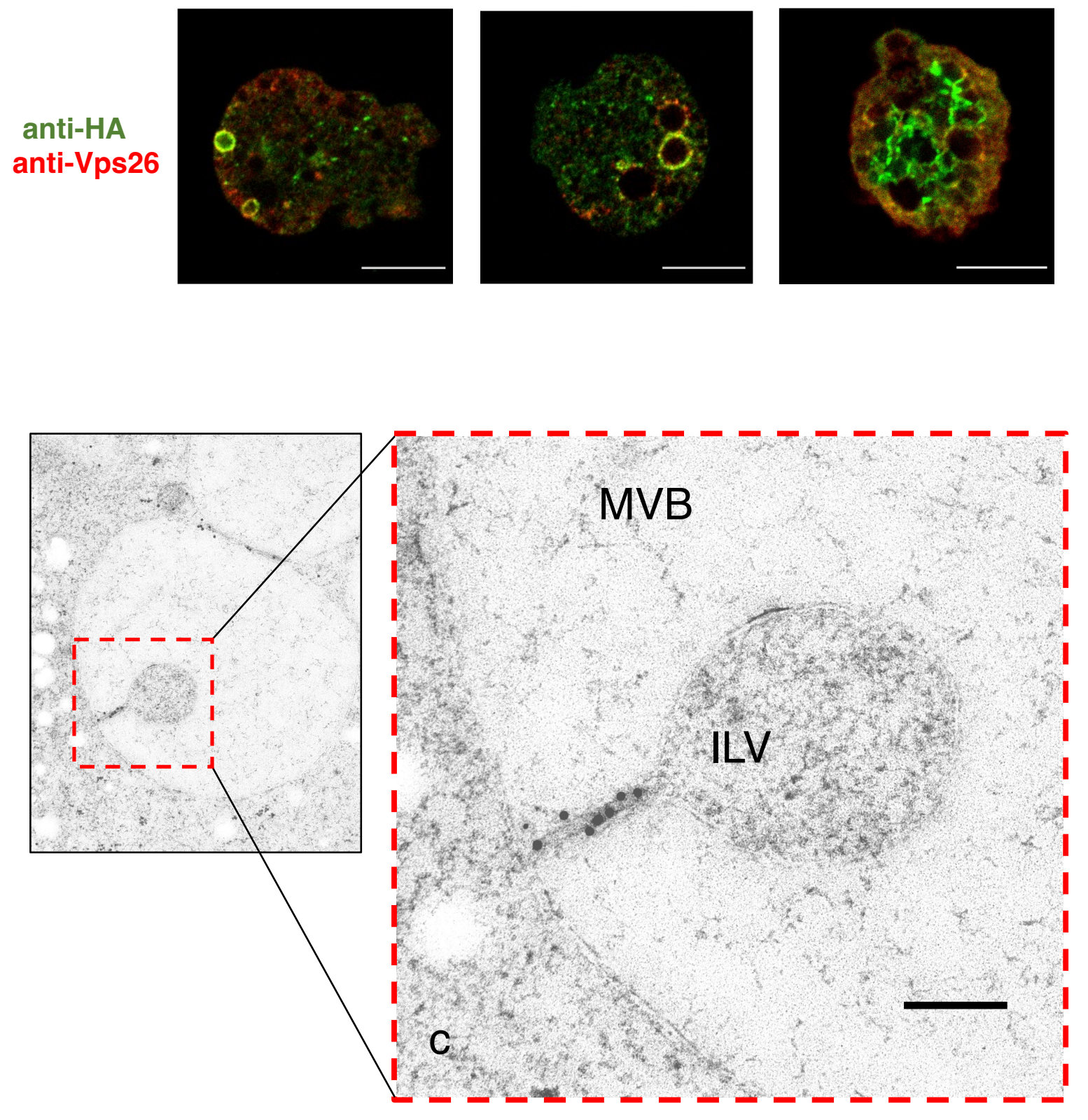

anti-Vps26 
Figure 8

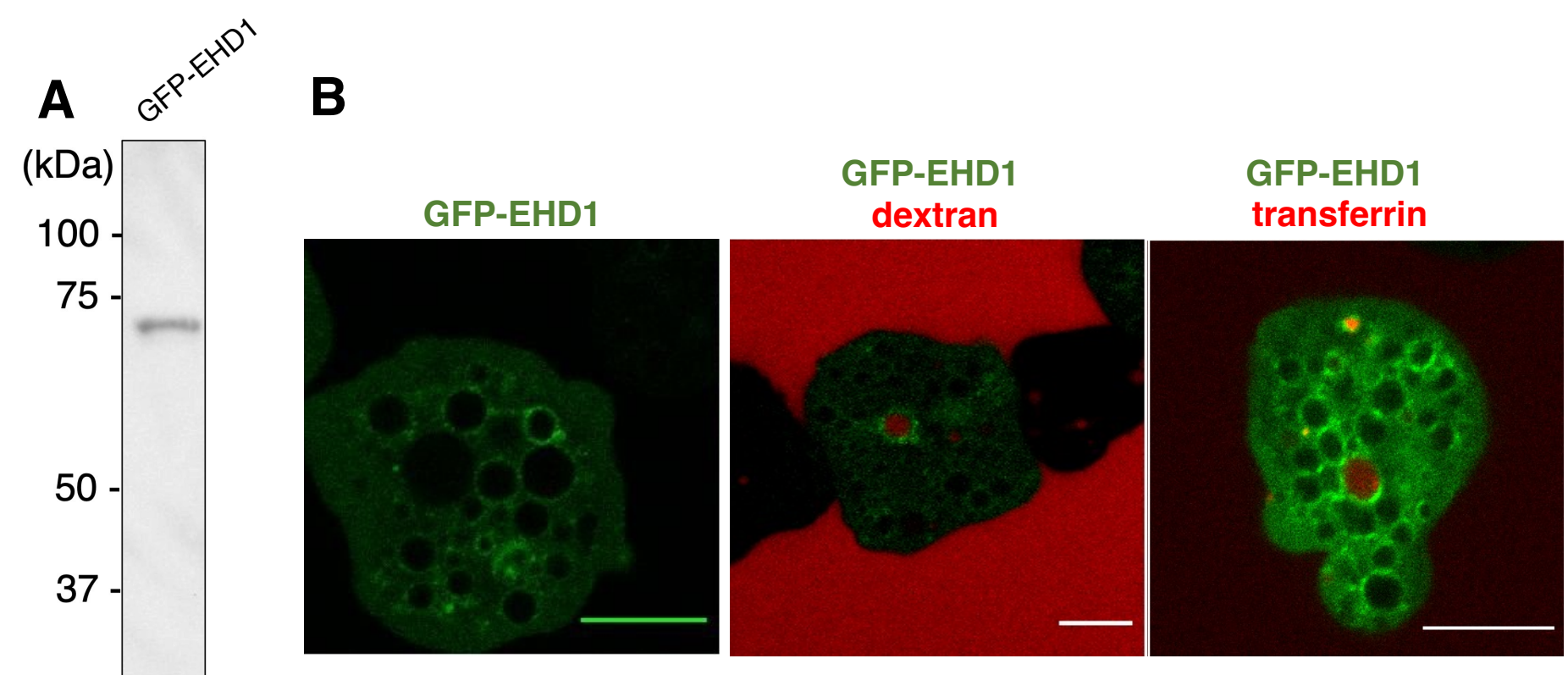

25 
